\title{
Unsteady Force Generation and Vortex Dynamics of Pitching and Plunging Flat Plates at Low Reynolds Number
}

\author{
Yeon Sik Baik ${ }^{1}$, Luis P. Bernal ${ }^{2}$, and Wei Shyy ${ }^{3}$ \\ University of Michigan, Ann Arbor, MI \\ Michael V. $\mathrm{Ol}^{4}$ \\ Air Force Research Lab, Wright-Patterson AFB
}

An experimental investigation of pitching and plunging flat plate at a prescribed effective angle of attack in the $S t$ range of 0.16 to 0.32 and $k$ range of 0.5 to 1.0 is presented. Force measurements and PIV technique were used to analyze and quantify the unsteady flow field created by the wing kinematics. A momentumbalance based non-intrusive force measurement technique and a systematic vortex detection algorithm were successfully implemented as a post-processing for PIV data to gain insight on the interplay between the unsteady force generation and vortex dynamics. It was determined that the inertial effects, which are proportional to the $S t$, dominate the unsteady force generation while the differences in the force generation for the same $S t$ kinematics are contributed by the circulatory effects such as the size and location of the leading edge vortex. The normal force with respect the flat plate was the primary contributor to the lift and drag production, and the projection of the normal force due to high pitch amplitude at higher $S t$ prompted higher thrust generation.

\section{Nomenclature}

\begin{tabular}{|c|c|}
\hline$\alpha$ & Geometric angle of attack [ $\left.\mathrm{rad}^{\circ}{ }^{\circ}\right]$ \\
\hline$\alpha_{0}$ & Mean angle of attack [ $\left.\mathrm{rad}^{\circ}{ }^{\circ}\right]$ \\
\hline$\alpha_{\text {eff }}(t)$ & Effective angle of attack [ $\left.\mathrm{rad}^{\circ}{ }^{\circ}\right]$ \\
\hline$\alpha_{\mathrm{plmax}}$ & Maximum plunge angle of attack [rad or $\left.{ }^{\circ}\right]$ \\
\hline$\Gamma$ & Circulation $\left[\mathrm{m}^{2} / \mathrm{s}\right]$ \\
\hline$\Gamma_{1}, \Gamma_{2}$ & Vortex core and boundary criteria \\
\hline$\theta_{0}$ & Pitch amplitude $\left[\operatorname{rad~or~}^{\circ}\right]$ \\
\hline$\dot{\theta}(t)$ & Pitch rate $[\mathrm{rad} / \mathrm{s}$ or $\% / \mathrm{s}]$ \\
\hline$v$ & Kinematic viscosity of water $\left[\mathrm{m}^{2} / \mathrm{s}\right]$ \\
\hline$\rho$ & Density of water $\left[\mathrm{kgm}^{-3}\right]$ \\
\hline$\phi$ & Phase lag between pitching and plunging motions [ $\left.\operatorname{rad}^{\circ}{ }^{\circ}\right]$ \\
\hline$\vec{\omega}$ & 3-component vorticity vector \\
\hline$b$ & Span length $[\mathrm{m}]$ \\
\hline$c$ & Chord length [m] \\
\hline
\end{tabular}

\footnotetext{
${ }^{1}$ Graduate Research Assistant, University of Michigan, Department of Aerospace Engineering, yeonb@umich.edu

${ }^{2}$ Associate Professor, University of Michigan, Department of Aerospace Engineering, lpb@umich.edu

${ }^{3}$ Chair Professor, University of Michigan, Department of Aerospace Engineering, weishyy @umich.edu

Hong Kong University of Science and Technology, Department of Mechanical Engineering

${ }^{4}$ Aerospace Engineer, Air Vehicles Directorate, Wright-Patterson AFB, Michael.Ol@ wpafb.af.mil
} 


\begin{tabular}{|c|c|}
\hline$c_{l}, c_{d}, c_{m}, c_{p}$ & Lift, drag, moment, and power coefficient \\
\hline$D$ & Drag force $[\mathrm{N}]$ \\
\hline$f$ & Motion frequency $\left[\mathrm{s}^{-1}\right.$ or $\left.\mathrm{Hz}\right]$ \\
\hline$F_{x}, F_{y}$ & Tangential and normal force component $[\mathrm{N}]$ \\
\hline$\vec{F}$ & 3-component force vector $[\mathrm{N}]$ \\
\hline$h$ & Plunge amplitude $[\mathrm{m}]$ \\
\hline$h_{0}$ & Normalized plunge amplitude, $h / c$ \\
\hline$h(t)$ & Plunge rate $[\mathrm{m} / \mathrm{s}]$ \\
\hline$k$ & Reduced frequency, $\pi f c / U_{\infty}$ \\
\hline$L$ & Lift force $[\mathrm{N}]$ \\
\hline$M$ & Total moment force $[\mathrm{Nm}]$ \\
\hline$M_{z}$ & Moment force about the half-chord location $[\mathrm{Nm}]$ \\
\hline$\vec{n}$ & 3-component unit normal vector \\
\hline$P$ & Power $[\mathrm{W}]$ \\
\hline $\operatorname{Re}$ & Reynolds number, $c U_{\infty} / v$ \\
\hline$S t$ & Strouhal number, $2 \pi f c h_{0} / U_{\infty}=2 k h_{0} / \pi$ \\
\hline$t$ & Time $[\mathrm{s}]$ \\
\hline$T$ & Period of motion, $1 / f[\mathrm{~s}]$ \\
\hline$\overline{\bar{T}}$ & Viscous tensor $\left[\mathrm{kgm}^{-1} \mathrm{~s}^{-1}\right]$ \\
\hline$\vec{u}$ & 3-component velocity vector $[\mathrm{m} / \mathrm{s}]$ \\
\hline$U_{\infty}$ & Free stream velocity $[\mathrm{m} / \mathrm{s}]$ \\
\hline$\vec{x}$ & 3-component position vector $[\mathrm{m}]$ \\
\hline
\end{tabular}

\section{Introduction}

The study of pitching and plunging airfoil has been the topic of interest by aerodynamicists and biologists as a suitable mechanism for micro air vehicles (MAV) which has a high potential for serving in surveillance and reconnaissance missions. Flapping wing mechanisms seen on birds and insects are thought to be more suitable than fixed wing configuration at small length scales as the aerodynamic characteristics are substantially affected by the low Reynolds number, $R e$ [1-3]. The attractiveness of the flapping wing configuration is the combined lift and propulsive mechanism which would allow MAVs to operate without a dedicated propulsive device. The flapping wing mechanism, however, imposes challenges that are unseen in fixed wing vehicles, namely the formation of large scale vortical structures, unsteady three-dimensional effects, separation and reattachment, and fluid structure interaction [4]. There have been many experimental and computational studies on pitching and plunging wing in periodic motion with simplifying assumptions to obtain fundamental understanding in unsteady aerodynamics [5]. In particular, the Strouhal number, $S t$, which characterizes the vortex dynamics of the wake and shedding behavior of vortices of a flapping motion is identified to be the relevant non-dimensional parameter [6]. 
This paper focuses on a rigid two-dimensional pitching and plunging wing with kinematics that produce large vortical structures. The amplitude and the frequency of the motion are chosen such that the force generated from these kinematics deviates from the classical theory of unsteady aerodynamics $[7,8]$. The unsteady aerodynamics of pitching and plunging wing first emerged in studies of flutter and helicopter blade aerodynamics [9]. The formation of a leading edge vortex (LEV) during dynamic stall results in an increase in lift coefficient well beyond the static stall value [9]. Although the reported dynamic stall occurs at Re much larger than relevant to MAV applications, similar vortical structures are found in many unsteady low Re flows. At low Re, the formation of LEV is believed to be critical in force generation in hovering insects through delayed stall by the LEV [10-16]. Shyy and Liu [17] reported that the LEV is common flow feature observed in flapping wing aerodynamics, but emphasized the fact that the characteristics and the effect on force generation can vary as Re, $k, S t$, wing flexibility, and kinematics are changed. While the unsteady aerodynamics of insects is three-dimensional in nature, large vortical structures are also reported in two-dimensional airfoil undergoing pitching and plunging motions $[18,19]$. Many two-dimensional pitching and plunging wing studies show that the wake patterns that produce thrust are governed by the $S t$ [20-24]. The commonly accepted form of $S t$ in pitching and plunging wing is defined as the ratio between the plunge amplitude multiplied by motion frequency, and the free stream velocity. The parameters involved in $S t$ are closely related to the effective angle of attack profile generated by the plunge motion but it does not include any information about the pitching motion. This imposes difficultly in analyzing the characteristic of pitching and plunging wing solely based on $S t$ as there can be multiple kinematics that share the same $S t$.

To better understand the role of $S t$ on pitching and plunging airfoils, a parametric study varying $S t, k$, and $\theta_{0}$ while preserving the effective angle of attack profile was performed by Baik et al. [25] The Re based on chord was at $R e=1 \times 10^{4}$ and a sinusoidal effective angle of attack profile with the minimum of $-6^{\circ}$ and maximum of $22^{\circ}$ was considered which led to the formation of a large LEV [26]. The motion frequency and amplitude were varied to achieve a $S t$ range from 0.1 to 0.2 . The LEV core trajectory in the frame of reference moving with the airfoil was measured and it was shown that reduced frequency was the dominant non-dimensional parameter controlling formation time of the LEV. The sinusoidal effective angle of attack profile was motivated by Read et al. [27] where it was reported that a sinusoidal effective angle of attack at higher $S t$ increases the thrust coefficient substantially compared to the effective angle of attack profile produced by a sinusoidal plunge motion. Hover et al. [28] expanded the work by performing a series of experiments with different airfoil kinematics with the effective angle of attack profiles consisting of a square wave, a symmetric sawtooth wave, a cosine wave, and sinusoidal wave plunge motion. It was concluded that the cosine wave effective angle of attack and the sinusoidal plunge motion produces the highest propulsive efficiency of $64 \%$, but the cosine wave profile produced nearly 4 times greater thrust coefficient. Moreover, Schouveiler et al. [29] investigated the effects of $S t$ and the maximum angle of attack on a harmonically flapping foil and reported high thrust and high efficiency. From these observations, it is strongly suggested that the plunge motion should be modified to produce the same effective angle of attack profile as the $S t$ is increased.

This paper extends the parametric study of pitching and plunging flat plates [25] by increasing $S t$ and $k$ ranges up to 0.32 and 1 , respectively. The main focus here is to explore the role of aggressive wing kinematics on vortical and inertia force producing mechanisms and their relative efficiency. A detail flow topology is reported by the 2D PIV with the unsteady force measurements acting on the flat plate. The $S t$ above 0.3 is of interest because many biological flyers operate at $S t$ between 0.25 and 0.35 [30], and this range of $S t$ is reported to produce optimum propulsive efficiency [31]. The Re based on chord for the present study is 5,000 and the effect of $R e$ is expected to be negligible due to separation at the leading edge of the flat plate produced by the effective angle of attack profile $[32,33]$. Two force measurement 
techniques are used in this study. Direct force measurement using a 6-component strain gauge balance provides the lift and drag forces as well as the pitching moment acting on the airfoil. The second approach is to use the "Flux Equation" proposed by Noca et al. [34] which uses PIV measurements of the velocity field and momentum balance to determine the force acting on an airfoil cross-section. This method is nonintrusive and attractive for the present study since the velocity field is already known from PIV measurement, which gives an independent measurement of sectional force coefficients. The vortex identification method proposed by Graftieaux et al. [35] was implemented as a post-processing procedure to provide detailed characterization of LEV location and size during the motion. The purpose of the LEV analysis is to identify the effects of LEV on the overall force generation.

\section{Methodology}

\section{A. Experimental setup}

The experiments are conducted at the University of Michigan low-turbulence water channel facility. The test section is 2-by-2 feet and is capable of generating free stream velocities ranging from $6 \mathrm{~cm} / \mathrm{s}$ to $40 \mathrm{~cm} / \mathrm{s}$. The free stream turbulence intensity at the free stream conditions of the present tests $(6 \mathrm{~cm} / \mathrm{s})$ was measured to be approximately $1 \%$. This value was computed by averaging 200 PIV images without the model in the test section. The turbulence intensity value of $1 \%$ is typical for low speed flows and the measurements were reproducible on different days. The turbulence level is much improved at higher free stream velocities with a reported value of order of $0.1 \%$.

The model used in current experiment is an infinite aspect ratio (AR) stainless steel flat plate wing with rounded leading and trailing edges. It has a $6.25 \%$ thickness to chord ratio with a chord length of 3 inches. An endplate was installed just below the free surface to minimize free surface effects and the distance between the wing and the bottom surface of the channel wall was kept at approximately $1 \mathrm{~mm}$. Figure 1 shows schematics of the water channel facility and the mounting setup. A 6-comopnent force sensor (ATI Industrial Mini40 model) was used for the direct force measurements. The sensor axis was placed at the half-chord location. For the present experiments, the pivot point of the airfoil pitching motion was the quarter-chord location. As shown in Figure 1, the model was attached in a cantilever fashion to the rotary stage (Velmex B4872TS) which in turn was attached to the linear traverse (Velmex BiSlide) such that pitching and plunging motion could be implemented. The motors are stepper motors and are controlled by a motor controller (Velmex VXM-1-1) which is connected to a computer. Each motor is controlled by specifying the speed and the displacement of the motor through a scripted computer code.

There are three main experimental techniques used in current study; dye flow visualization, 2D particle image velocimetry (PIV), and direct force measurement. Dye flow visualization was used for qualitative and exploratory study of pitching and plunging wing kinematics. The flow visualization system consists of a dye rake, two syringe pumps, 2 syringes, and food coloring dye. The dye rake consists of seven uniformly spaced dye streams spaced 1-inche apart placed approximately one chord length upstream of the airfoil. The 1-inch spacing between the dye streams is adequate since the amplitude of the airfoil plunging motion does not exceed 6 inches. The flow visualization is simple, yet a powerful technique to identify large scale structures in the flow field which also serves as a qualitative

validation for a PIV data set acquired for the same conditions. A more detailed description of dye injection system can be found in Baik et al. [25] 

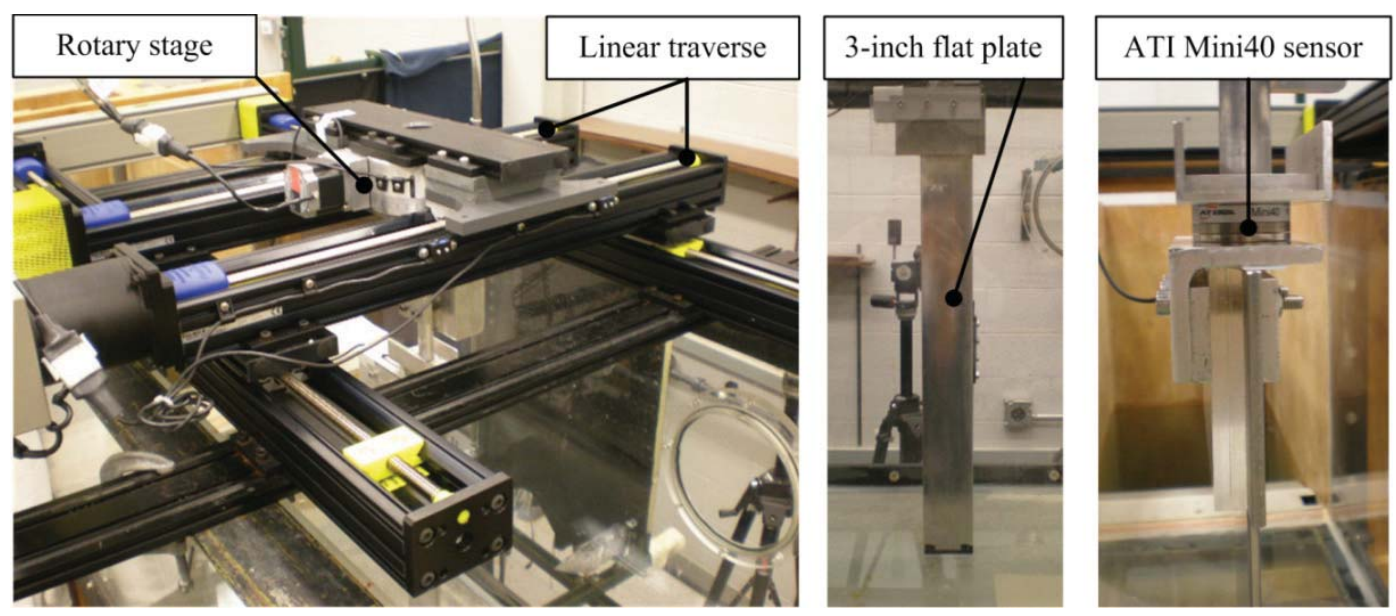

Figure 1. Water channel facility and the experimental setup at the University of Michigan.

The PIV system consists of a double-pulsed Nd-YAG laser (Spectra Physics PIV 300), light sheet formation optics, a mechanical shutter, a dual frame digital camera (Cooke Corp. PCO.4000), a Nikon 105-mm Micro-Nikkor lens, computer image acquisition system, a signal generator, and a microcontroller based synchronization electronics. The water channel was seeded with $3 \mu \mathrm{m}$ diameter Titanium Dioxide particles (Sigma-Aldrich) and a small amount (8 drops) of a dispersant (DARVAN C-N, Vanderbilt) was used to produce a uniform distribution of particles and to help maintain the particles in suspension for the duration of experiment. The goal is to acquire phase-locked 2D PIV images such that phase-averaging can be performed at the post-processing stage. The synchronization electronics is responsible for synchronizing the laser trigger, the laser shutter, and the camera, with the model motor controller in order to produce a phase-locked PIV images with consistency. The drift in airfoil position measured after 50 cycles was approximately $1 \sim 2$ pixels, which is negligible in the data analysis. The magnification for the present experiments is 12 pixels $/ \mathrm{mm}$ and the camera field of view is $334 \mathrm{~mm}$-by$222 \mathrm{~mm}$, which corresponds to the 4008-by-2672 pixels frame size of the PCO-4000. This field of view successfully captured approximately 4 chord lengths in the free stream direction and 3 chord lengths in the plunge motion direction. The time between image pairs was adjusted to give 6 pixels displacement at free stream velocity. A typical experiment consists of 55 motion cycles where the first 5 cycles are discarded to neglect initial transient effects. A total of 100 PIV image pairs were acquired for each phase of the motion by repeating the experiment twice. After the data acquisition, the images were analyzed using in-house developed MATLAB software. The software filters the data, removes the outliers, and performs statistical averages to output phase-averaged results. A detailed description of the PIV postprocessing software can be found in Baik et al. [25]

The direct force measurement system consists of a force/torque sensor (ATI industrial automation Mini40), interface power supply (ATI industrial automation 9105-IFPS-1), a data acquisition card (National Instrument PCI-6625), and a computer. Attachment of the force/torque sensor to the model is shown in Figure 1. The Mini40 is a 6-component silicon-strain-gauges based sensor capable of measuring forces in the plane of the airfoil cross section up to $\pm 80 \mathrm{~N}$ and $\pm 240 \mathrm{~N}$ in the orthogonal direction. It also measures torque up to $\pm 4 \mathrm{Nm}$ in all 3 axis. The resolution is $1 / 50 \mathrm{~N}$ for force and 1/2000 $\mathrm{Nm}$ for torque. The reference axes convention of the force/torque sensor readings is shown in Figure 2. 


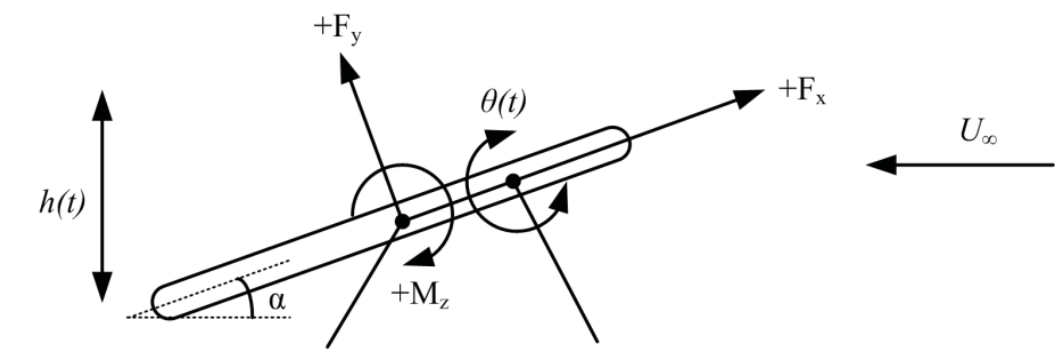

Half-chord (Force measurement location) Quatrter-chord (Pivot location)

Figure 2. A schematic of sign convention used for the force/torque sensor readings.

$\mathrm{F}_{\mathrm{y}}$ is the normal force, $\mathrm{F}_{\mathrm{x}}$ is the tangential (chordwise) force, $\mathrm{M}_{\mathrm{z}}$ is the torque of the model, $U_{\infty}$ is the free stream velocity, $\alpha$ is the geometric angle of attack, $\theta(t)$ is the pitch angle, and $h(t)$ is the plunge displacement. The value of lift, drag, and moment is derived from the measured forces and the geometric angle of attack and it is shown in Eq. (1).

$$
\begin{aligned}
& L=F_{x} \sin (\alpha)+F_{y} \cos (\alpha) \\
& D=-F_{x} \cos (\alpha)+F_{y} \sin (\alpha) \\
& M=M_{z}+0.25 c F_{y}
\end{aligned}
$$

The second term in the moment calculation is introduced because that the pivot location is located at the quarter-chord location and the force/torque sensor axis is located at the half-chord location. The symbol $c$ denotes the chord length. The power is also computed through knowledge of the prescribed pitching and plunging motion and it is shown in Eq. (2). $\dot{h}(t)$ is the plunge velocity and $\dot{\theta}(t)$ is the pitching rate in $\mathrm{rad} / \mathrm{s}$.

$$
P=L \dot{h}(t)+M \dot{\theta}(t)
$$

The power is used to compute the thrust efficiency, $\eta_{T}$, which is the ratio of the thrust and power coefficients. The equations for computing the lift, drag, moment, and power coefficients are summarized in Eq. (3). $\rho$ is the density of water, $c$ is the chord length, $b$ is the span of the wing, and $U_{\infty}$ is the free stream speed.

$$
c_{l}=\frac{L}{0.5 \rho c b U_{\infty}^{2}}, c_{d}=\frac{D}{0.5 \rho c b U_{\infty}^{2}}, c_{m}=\frac{M}{0.5 \rho c^{2} b U_{\infty}^{2}}, c_{p}=\frac{P}{0.5 \rho c b U_{\infty}^{3}}
$$

Two different tests were performed to obtain time history of force coefficients: a tare test and a force test. The tare test was performed in air to measure the inertial load on the force/torque sensor; the water level was lowered in the test section to perform the tare test. After the tare test, the water level was raised to the span of the wing model and the force test was performed. The tare test results were then subtracted from the force test results to obtain hydrodynamic loading on the wing model.

Similar to the PIV data acquisition, the force measurements were phase-averaged over 500 motion cycles for each wing kinematic. A typical force measurement experiment consisted of 110 measurements with 5 seconds of pre-trigger data. The purpose of pre-trigger data was to eliminate the bias readings as the raw voltage readings with sensor at rest position varied in time. The oscillation in the raw voltage 
readings after conversion to forces was documented to be within $\pm 0.05 \mathrm{~N}$ with a period of approximately 2 hours. The running time of a typical experiment with the longest period of approximately 7.2 seconds is 13 minutes, which results in $0.01 \mathrm{~N}$ bias between the first cycle and the last cycle within the data set. However, this bias had a negligible impact on the phase-averaged data set due to the large number of samples with measurements exceeding order of $0.01 \mathrm{~N}$. The first 5 and the last 5 cycles were discarded for two reasons. Firstly, discarding the first 5 cycles is consistent with the elimination of the initial transient effect used in PIV acquisition. Secondly, the force data were low-pass filtered using a sharpfrequency-cut-off Fourier filter which introduced significant initial and end transients lasting approximately three cycles. The force/torque sensor electronics did not include an analog filter to remove EMI and other analog noise. In order to remove the analog noise, a sampling frequency above $1000 \mathrm{~Hz}$ was needed to capture the broadband analog noise which was later removed by the low-pass filter. All the data sets were sampled at $2000 \mathrm{~Hz}$ and low-pass filtered with a cutoff frequency of $1 \mathrm{~Hz}$. The filter cutoff frequency was chosen to remove force sensor signal associated with structural vibration of the cantilevered wing, which was at approximately $6 \mathrm{~Hz}$ and the noise was still present in the data at $2 \mathrm{~Hz}$ filter cutoff frequency.

\section{B. Noca's Flux Equation (FE)}

For the indirect force measurement, the "Flux Equation" (FE) developed by Noca et al. [34] was implemented. It is a momentum-balance approach and the formulation allows the force coefficients to be calculated from the measured velocity vector fields and their time derivatives, which are readily available from PIV data. Noca et al .[34] developed several formula to compute the force acting on a body given an arbitrary control volume that encloses the body. In particular, the FE only requires a surface integral whereas the other formulas require a volume integral over the control volume. The FE is given below where $S(t)$ and $S_{b}(t)$ denotes arbitrary control volume enclosing the body and body boundary, respectively.

$$
\begin{gathered}
\vec{F}=\oint_{S(t)} \vec{n} \cdot \gamma_{\text {flux }} d S-\oint_{S_{b}(t)} \vec{n} \cdot\left(\vec{u}-\vec{u}_{s}\right) d S-\frac{d}{d t} \oint_{S_{b}(t)} \vec{n} \cdot(\vec{u} \vec{x}) d S \\
\gamma_{\text {flux }}=\frac{1}{2} u^{2} \bar{I}-\vec{u} \vec{u}+\frac{1}{N-1}(\vec{\omega}(\vec{x} \times \vec{u})-\vec{u}(\vec{x} \times \vec{\omega})) \\
-\frac{1}{N-1}\left[\left(\vec{x} \cdot \frac{d \vec{u}}{d t}\right) \bar{I}-\vec{x} \frac{d \vec{u}}{d t}+(N-1) \frac{d \vec{u}}{d t} \vec{x}\right] \\
+\frac{1}{N-1}[\vec{x} \cdot(\nabla \cdot \overline{\bar{T}}) \overline{\bar{I}}-\vec{x}(\nabla \cdot \bar{T})]+\overline{\bar{T}}
\end{gathered}
$$

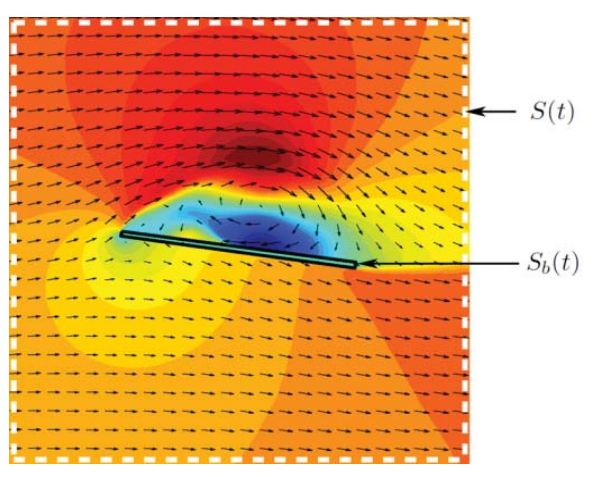

$\vec{n}$ is the unit normal 3-component vector, $\vec{u}$ is the 3-component velocity vector, $\vec{x}$ is the 3-component position vector, $\overline{\bar{I}}$ is a unit matrix or identity matrix, $\overline{\bar{T}}$ is the viscous tensor, and $N$ is the dimension. The $2^{\text {nd }}$ term in the FE is equal to zero because of no slip condition at the surface of the body. The $3^{\text {rd }}$ term can be ignored because the thickness of the airfoil is small. Therefore, only the information on the surface of the control volume enclosing the body is needed to obtain the force coefficients. Compared to other formulations introduced by Noca et al. [34], the FE circumvents the need for volume integrals including 
the velocity field near the body surface which are difficult to measure in experiments. The FE assumes that the flow is divergence free which is satisfied for incompressible flow. However, if the PIV-measured flow field is not entirely divergence free it may be a possible source of error in the results.

It was documented from numerical and experimental data that the FE is sensitive to the origin and control volume locations. It is mentioned in Noca et al. [34] that the computed force coefficient is larger in magnitude if the control volume is too small i.e. too close to the airfoil. It is also apparent from his results that the force coefficient value changes as the origin is varied. In order to investigate these problems, each term in the FE was carefully examined. By gathering the terms proportional to the position vector, $\vec{x}$, it can be shown that the FE is independent of the origin location when the rate of change of circulation equals to the net flux of vorticity in the control volume, as shown in Eq. (4). In order for this equality to hold at different control volume and origin locations, the spatial and time derivatives will both have to be accurate. To test this hypothesis, the force coefficients using FE was computed for 11 different control volumes with 25 uniformly scattered origin locations at each control volume. Figure 3 illustrates the control volume and origin selections.

$$
\frac{d \Gamma}{d t}=\oint \frac{\partial \vec{u}}{\partial t} \cdot d \vec{l}=-\oint \omega_{z}(\vec{u} \cdot \vec{n}) d S
$$

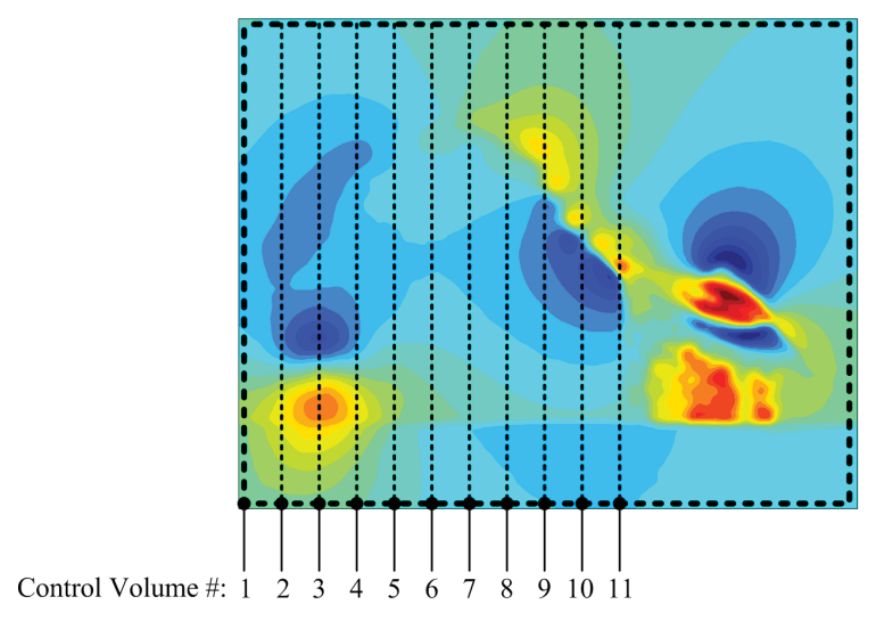

(a)

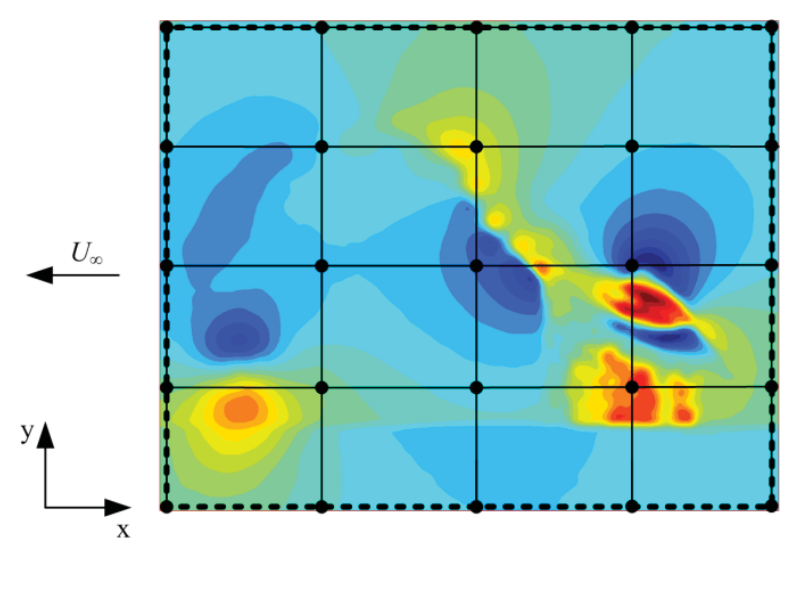

(b)

Figure 3. (a) A schematic of different control volume used to test the control volume and origin independence in FE, and (b) an illustration of 25 uniformly scattered origins for a given control volume.

A central time differencing scheme was implemented to compute the time derivates of velocity vectors which requires 3 successive images in time. The control volume must be the same for the 3 images used in order to apply FE successfully. Control volume \#1 has the largest field of view which captures approximately 3.5 chord lengths downstream, and control volume \#11 has the smallest field of view with approximately 0.5 chord lengths downstream. A total of 25 origins were uniformly scattered within the control volume as shown in Figure 3(b). This resulted in 25 different force coefficients and they were averaged to compute the mean force coefficients for a given control volume. For the present measurements, the PIV light sheet entered the test section on one side which resulted in a shadow region behind the airfoil in the opposite direction. The shadowed region is easily noticeable by irregular contours present in the flow field just below where the airfoil is present. To obtain data in the shadow region the experiments were repeated with the laser sheet entering the test section on the other side of the airfoil. These experiments were conducted on the same day to minimize errors. The resulting two sets of PIV 
images were used to compute phase averages and the phased-averaged PIV data were stitched together to complete the entire closed contour around the airfoil. However, it is shown in Figure 3 that the stitching is not perfect; there are some discontinuities present between the top and bottom where the stitching occurs. Since the FE requires information on the control surface that surrounds the body, approximately $1 / 8$ of the bottom image was stitched in order to overcome the shadowed region in the flow field. The remaining shadowed region in the middle of the image did not affect the FE calculations. The effect of the discontinuity has not been explored. The only feasible way to avoid stitching of the images for FE application is to acquire data with dual laser sheet, and this is an option for future experimental efforts.

Numerous calculations were performed for different control volumes: circulation, rate of change of circulation, lift coefficient, and drag coefficient from FE methodology. Figure 4 summarizes these results for the flow field shown in Figure 3. There are two ways to compute circulation: closed integral around the control surface involving velocity vectors, and summation of $\omega_{z}$ component in the control volume. This comparison verifies the accuracy of the computed vorticity, and Figure 4(a) shows good agreement between the two methods used to compute circulation. A slight discrepancy was found at control volume \#3 through \#5, and this is due to a discontinuity in the stitching region where strong vorticity is present. Figure 4(b) assesses the control volume and origin independence in FE by calculating the rate of change of circulation from Eq. (4). The difference, which is shown in circles, should be zero if FE is independent of origins. The results show that either the time rate of change of the velocity vector is under-predicted, or the $\omega_{z}$ flux is over-predicted. Both methods require numerical integration along the control surface and considering that the magnitude of $\omega_{z}$ is an order of magnitude higher than the time rate of change of velocity, a significant numerical integration error could be present. On the other hand, if the time increment between the images is too large, the rate of change of velocity can suffer from inaccuracy where large vortical structures are present. This explains why there are large discrepancies present at control volumes \#3 through \#5 where a large vortical structure introduces large vorticity magnitude and velocity gradients along the control surface. The best results were obtained at control volumes \#5, \#8, and \#9 and the results for the lift and drag coefficients are shown in Figure 4(c) and (d). The values of lift and drag coefficients are similar and the errors originating from different origin locations are small compared to other control volumes. The error bars denote $95 \%$ confidence interval for 25 different force coefficients computed at different origins. The average of origin and control volume independent values is reported as the lift and drag coefficients at each phase of the motion in the 'Results' section of this paper.

\section{Vortex identification method}

The main focus of vorticity analysis dynamics in current study is to determine the evolution of the LEV since it is believed to have a critical role in generating high lift in unsteady aerodynamics. It involves two steps: the detection of the vortex core and the analysis of its strength by computing the circulation from the vortex boundary. A popular method of identifying the vortex core and its boundary is to perform point-wise analysis of the velocity gradient tensor [36]. However, the main drawback of such method is that it computes multiple vortex cores within an enclosed vortex boundary due to small-scale turbulence present within the vortex [25]. Vortex identification method used by Graftieaux et al. [35] overcomes this problem by introducing 2 scalar functions derived from the velocity vector fields which identify the LEV core locations and its boundary; $\Gamma_{1}$ criterion identifies the vortex core, and $\Gamma_{2}$ criterion identifies the boundary of a vortex. $\Gamma_{1}$ and $\Gamma_{2}$ criteria are given by Eq. (5) and (6), respectively. 


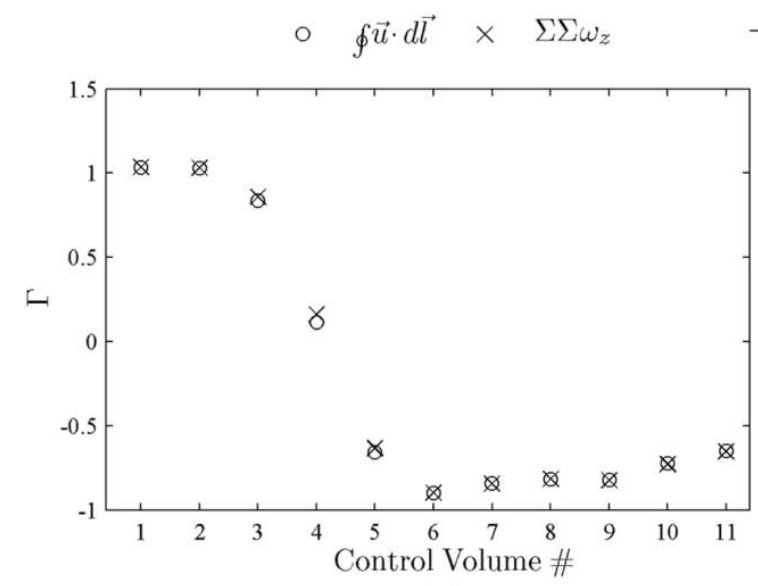

(a)

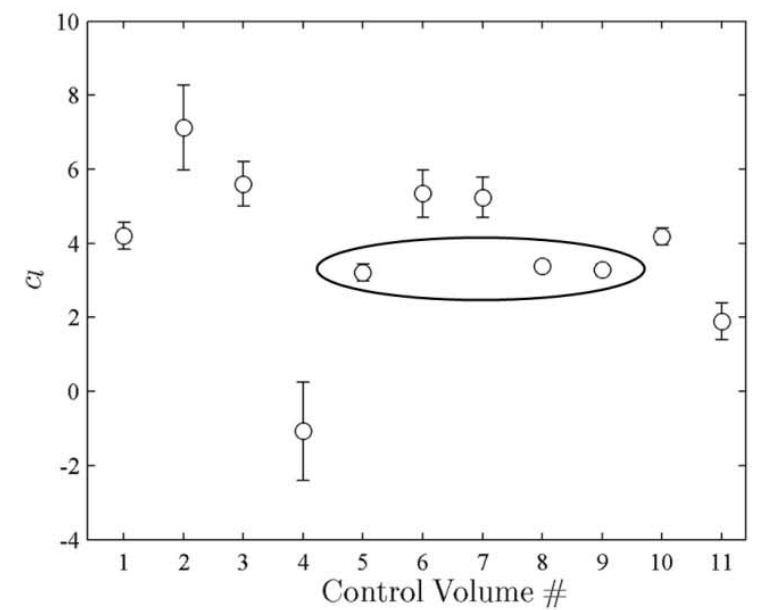

(c)

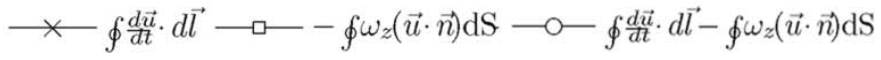

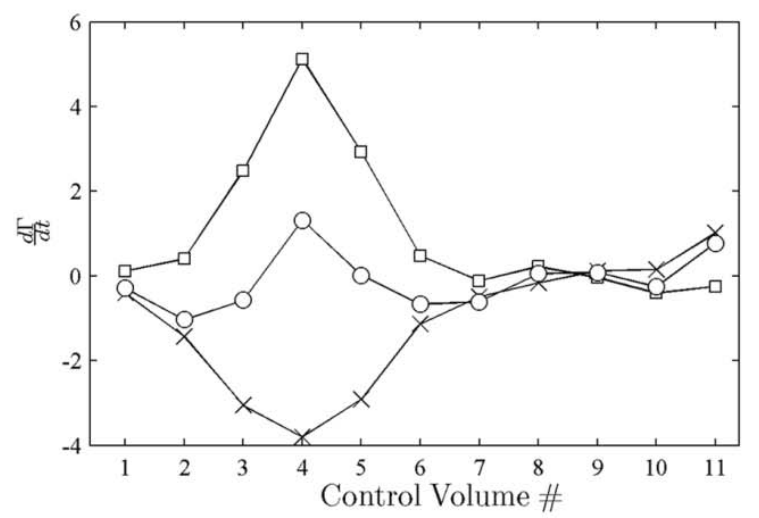

(b)

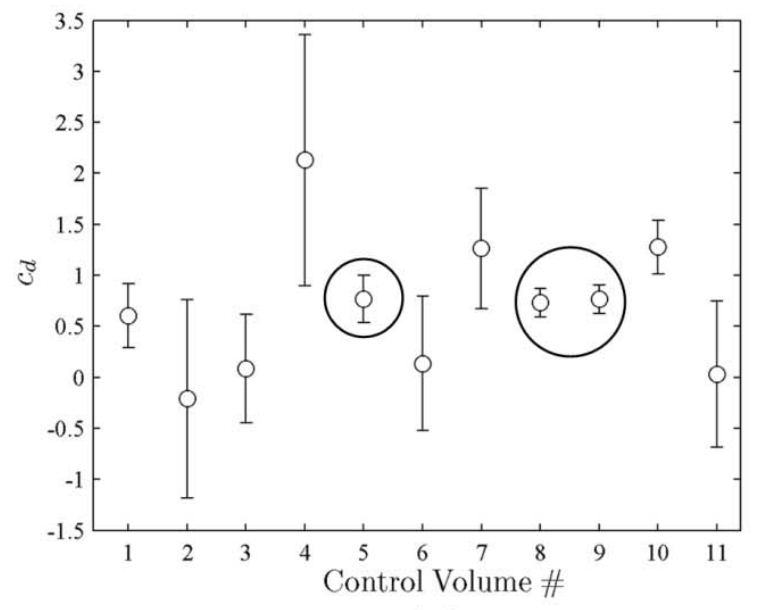

(d)

Figure 4. For a different control volume the following were computed: (a) circulation calculation involving the velocity on the control surface and summation of $\omega_{z}$ in the control volume, (b) rate of change of circulation calculation involving the time rate of change of velocity around the control surface and $\omega_{z}$ flux, (c) lift coefficient from FE, and (d) drag coefficient from FE.

$$
\begin{gathered}
\Gamma_{1}(p)=\frac{1}{N} \sum_{i=1}^{N} \frac{\left(\left(\overrightarrow{x_{p}}-\overrightarrow{x_{i}}\right) \times \overrightarrow{u_{i}}\right) \cdot \vec{n}}{\left\|\overrightarrow{x_{p}}-\overrightarrow{x_{i}}\right\| \cdot\left\|\overrightarrow{u_{i}}\right\|} \\
\Gamma_{2}(p)=\frac{1}{N} \sum_{i=1}^{N} \frac{\left(\left(\overrightarrow{x_{p}}-\overrightarrow{x_{i}}\right) \times\left(\overrightarrow{u_{i}}-\overrightarrow{u_{p}}\right)\right) \cdot \vec{n}}{\left\|\overrightarrow{x_{p}}-\overrightarrow{x_{i}}\right\| \cdot\left\|\overrightarrow{u_{i}}-\overrightarrow{u_{p}}\right\|}
\end{gathered}
$$

$p$ is any point on the flow field, $\vec{x}$ is the position vector, $\vec{u}$ is the velocity vector, $\vec{n}$ is the unit vector in the $z$-direction, and $N$ is the total number of points in a control volume. The algorithm selects $N$ number of points around a point $P$ and computes $\Gamma_{1}$ and $\Gamma_{2}$. A total of 9 points were used in a control volume for current study and $\overline{u_{p}}$ is the average velocity at point $P$ computed from surrounding 9 points. The maximum value of $\Gamma_{1}$ and $\Gamma_{2}$ is 1 by construction. A vortex core typically has a $\left|\Gamma_{1}\right|$ value greater than 0.9 . 
$\Gamma_{2}$ criterion determines whether the flow at point $P$ is dominated by strain or rotation; if $\left|\Gamma_{2}\right|$ is less than $2 / \pi$ it is strain dominated and if $\left|\Gamma_{2}\right|$ is greater than $2 / \pi$ it is rotation dominated. It is possible to have a rotating flow with no definite vortex core but still have $\left|\Gamma_{2}\right|$ greater than $2 / \pi$. While the theoretical threshold value of $\left|\Gamma_{2}\right|$ for rotation dominated flow is $2 / \pi$, the method is weakly dependent on the distance between points and a characteristic length scale of the vortex. Graftieaux et al. [35] showed that the threshold value of $\left|\Gamma_{2}\right|$ stays within the range of $0.6 \sim 0.7$ regardless of the ratio of characteristic lengths. The processed PIV data have a set distance between points of 16 pixels and $\left|\Gamma_{2}\right|$ threshold value was set at 0.65. A sample plot of vortex identification algorithm is shown in Figure 5.

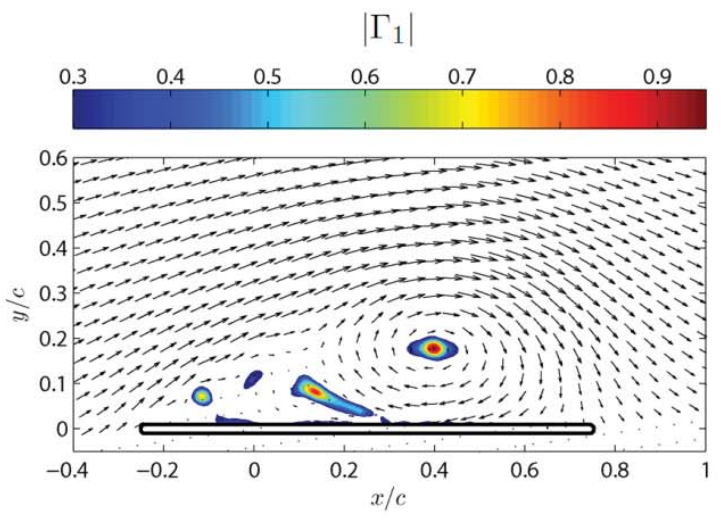

(a)

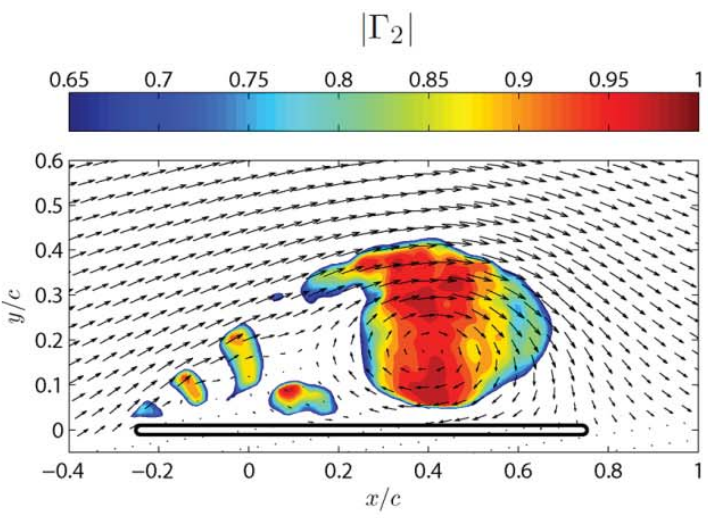

(b)

Figure 5 The velocity vectors with (a) contour of $\left|\Gamma_{1}\right|$, and (b) contour of $\left|\Gamma_{2}\right|$

Figure 5(a) shows a vortex core at $x / c \approx 0.4$ which is in excellent agreement with the velocity vectors. At $x / c \approx 0.15$, a $\left|\Gamma_{1}\right|$ value of 0.85 is recorded which indicates the possibility of secondary vortex formation. The boundary of the vortex core located $x / c \approx 0.4$ is shown in Figure 5(b). Notice that there are 3 distinct regions captured by the $\left|\Gamma_{2}\right|$ contours in the shear layer location. This flow region is rotation dominated but it does not have a definite vortex core. The boundary of the LEV enables the computation of its circulation through Eq. (7), where $\Gamma$ is the circulation.

$$
\Gamma=\iint \vec{\omega} \cdot \vec{n} d A=\sum_{i=1}^{N} \omega_{z_{i}}
$$

The enclosed area of the LEV found from $\left|\Gamma_{2}\right|$ criterion consists of $N$ number of points in the PIV grid and the integral simplifies to summation of all the vorticity with the normal in the in-plane direction. The number $N$ is also used to compute the LEV cross section area.

\section{Results}

This paper aims to highlight the features in unsteady force generation in pitching and plunging flat plate by considering a specific airfoil kinematic, namely a kinematic that preserves a sinusoidal effective angle of attack. The equations used to generate the kinematics are presented along with chosen cases with governing non-dimensional parameters. There are a total of 3 cases with $k$ ranging from 0.5 and 1.0 , and St ranging from 0.16 and 0.32. Initially, the PIV and force measurements for each case are presented in detail. Then, the force measurements are compared with FE to gain insight on different components of the 
force contributing to the overall force acting on the flat plate. Lastly, the vortex dynamics associated with these cases and force measurement comparison is discussed to draw meaningful conclusions.

\section{A. Airfoil kinematics}

The wing motion kinematics considered in this paper is a pitching and plunging motion that produces a sinusoidal effective angle of attack profile with pivot location at the quarter-chord. A sinusoidal effective angle of attack, $\alpha_{\text {eff }}(t)$, is produced by the enforcing a sinusoidal effective angle of attack produced by the plunge motion, as shown in Eq. (8).

$$
\alpha_{\text {eff }}(t)=\alpha_{0}+\theta_{0} \cos (2 \pi f t+\phi)+\alpha_{\text {plmax }} \sin (2 \pi f t)=\alpha_{0}+\alpha_{\text {pitch }}+\alpha_{\text {plunge }}
$$

$\alpha_{0}$ is the mean angle of attack, $\theta_{0}$ is the pitch amplitude, $f$ is the motion frequency, $\phi$ is the phase lag between the pitching and plunging motion, and $\alpha_{\text {plmax }}$ is the maximum angle of attack produced by the plunging motion. In this equation, the plunging motion is unknown and it must be obtained by integrating Eq. (9), which is derived from the angle of attack produced by the free stream and the plunge directions.

$$
\operatorname{atan}\left(-\frac{\dot{h}(t)}{U_{\infty}}\right)=\alpha_{\mathrm{plmax}} \sin (2 \pi f t)
$$

$\dot{h}(t)$ is the velocity of plunging motion, and $U_{\infty}$ is the free stream velocity. After some rearrangements, the ordinary differential equation for the plunge motion is given by Eq. (10).

$$
\dot{h}(t)=-U_{\infty} \tan \left(\alpha_{\mathrm{plmax}} \sin (2 \pi f t)\right)=h_{0} c \dot{F}(t)
$$

$h_{\mathrm{o}}$ is the normalized plunge amplitude, typically normalized by the chord length, $c$. Since the plunging motion amplitude equals $h_{\mathrm{o}} c$, it follows that the $F$ must satisfy the integral condition shown in Eq. (11).

$$
\int_{0}^{0.25 f} \dot{F}(t) d t=\int_{0}^{0.25 f}-\frac{U_{\infty}}{h_{0} c} \tan \left(\alpha_{\text {plmax }} \sin (2 \pi f t)\right) d t=-1
$$

Regardless of the motion trajectory, the model must travel displacement equal to one amplitude every quarter-chord. $F(t)$ is found numerically through an iterative procedure where the value of $\alpha_{\text {plmax }}$ is assumed initially. It is determined that the value of $\alpha_{\mathrm{plmax}}$ is a function of $S t$, and this also implies that the pitch amplitude, $\theta_{0}$, is a function of $S t$. The $S t$ for current study is defined in Eq. (12).

$$
S t=\frac{2 \pi f c h_{0}}{U_{\infty}}=\frac{2 k h_{0}}{\pi}
$$

The plunge motion for sinusoidal effective angle of attack is not sinusoidal for St greater than approximately 0.1. For sinusoidal plunge motion with $S t$ greater than 0.1 , higher harmonics are 
introduced in the effective angle of attack time profile. Figure 6 illustrates the difference between sinusoidal motion and sinusoidal effective angle of attack producing plunging motion at $S t=0.32$. The difference in plunging motion is subtle between two cases but the resulting effective angle of attack profile differs greatly. The maximum discrepancy in effective angle of attack is approximately $6^{\circ}$ and there exists a double peak for the sinusoidal motion on the effective angle of attack. These double peak phenomena is observed at $S t$ greater than 0.2 and it becomes more apparent as $S t$ increases.

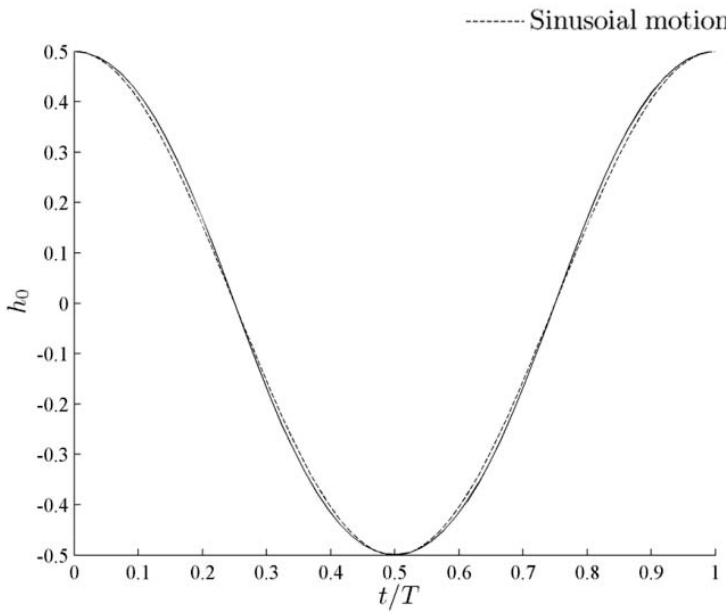

(a)

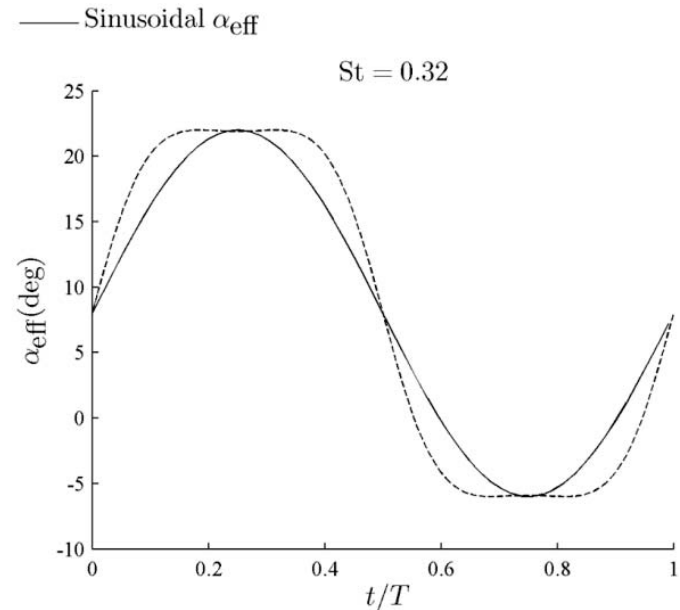

(b)

Figure 6. a) Difference in plunging motion for sinusoidal motion and sinusoidal effective angle of attack producing motion, and b) difference in effective angle of attack profile for two different motions at $S t=0.32$.

The effective angle of attack profile used in current study ranges between $-6^{\circ}$ and $22^{\circ}$, and this particular profile has been explored in many studies $[25,26,32,33]$. The initial angle of attack is set at $8^{\circ}$ and the angular amplitude considered is $\pm 14^{\circ}$. The phase lag between the pitching and plunging motion is fixed at $\phi=\pi / 2$ and the Re based on chord is kept at 5,000. The profile is shown in Figure 6(b). In order to achieve different reduced frequency, the motion frequency was varied such that $S t$ value up to 0.32 was achievable. The targeted non-dimensional parameters space for the current study is best presented in the $k-h_{0}$ space as shown in Figure 7.

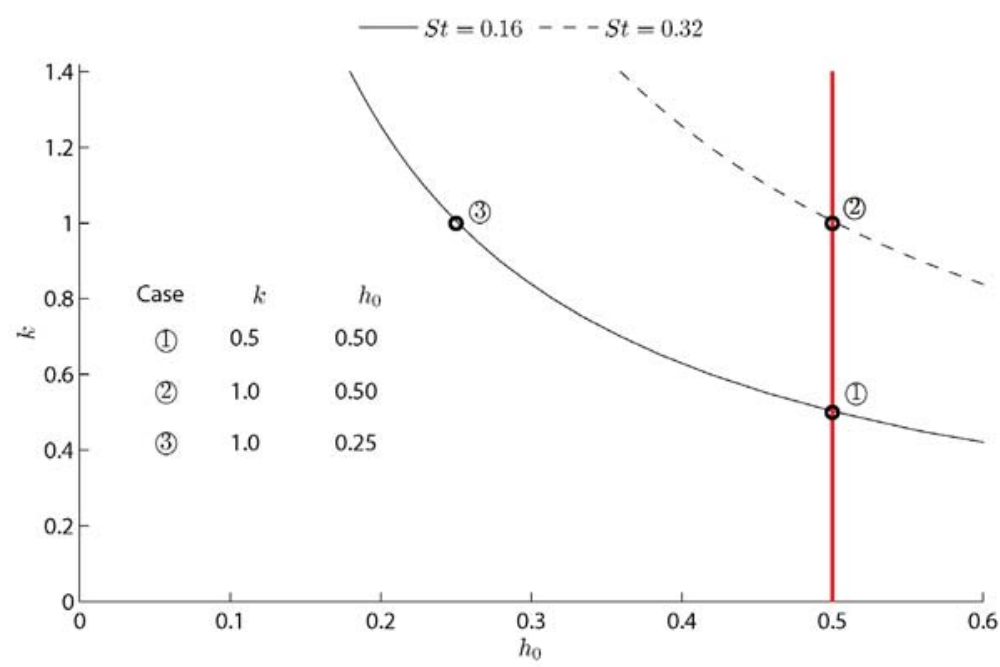

Figure 7. $k-h_{0}$ space depicting high and low reduced frequency cases. 
There are a total of 3 cases and corresponding non-dimensional parameters are shown in the figure as well as in Table 1. Cases 1 and 2 have constant $h_{0}$ of 0.5 and $S t$ is varied by altering $k$ from 0.5 to 1.0. For cases 2 and 3, the reduced frequency is fixed at $k=1.0$. Baik et al. [25] reported similarities in the flow structures with respect to the airfoil for the same $k$ value with the highest reported $k$ value of 0.63 and $S t=$ 0.2. The expectation from current cases is that the delayed formation of the LEV will still be present in the flow field even at $k=1.0$ with $S t=0.32$. Notice that the pitch amplitude for $S t=0.32$ is at $33.7^{\circ}$ which results in maximum and minimum geometric angle of attack of $41.7^{\circ}$ and $-25.7^{\circ}$, respectively. One of the main objectives of the present research is determine if the flow structure observed at lower $k$ cases are observed at higher $k$ value with high geometric angle of attack.

\begin{tabular}{cccccc} 
Case & $S t$ & $k$ & $h_{0}$ & $\left|\theta_{0}\right|\left(^{\circ}\right)$ & $T(\mathrm{~s})$ \\
\hline 1 & 0.16 & 0.5 & 0.50 & 13.16 & 7.14 \\
2 & 0.32 & 1.0 & 0.50 & 33.73 & 3.57 \\
3 & 0.16 & 0.5 & 0.25 & 13.16 & 3.57
\end{tabular}

Table 1. Summary of parameters used in current study.

B. $\quad$ Case $1(k=0.5, S t=0.16)$

Flow visualization and PIV measurements were taken at every $30^{\circ}$ phase, resulting in data set for $t / T$ $=0.000,0.083,0.167,0.250,0.333,0.417,0.500,0.583,0.667,0.750,0.833$, and 0.917. Figure A1 (Appendix A) summarizes the flow visualization, normalized $\mathrm{x}$-component velocity $\left(\bar{u} / U_{\infty}\right)$ contours, normalized vorticity contours $\left(\omega c / U_{\infty}\right)$, and vortex boundary $\left(\Gamma_{2}\right)$ during the down stroke of the cycle, namely $t / T=0.00$ to 0.50 . Figure A2 summarizes the same data set for the up stroke, namely $t / T=0.500$ to 0.917 . The flow topology resulting from case 1 kinematic is similar to past experiments conducted at different $k$-St combination and $R e$ for a flat plate [25]. The formation of a large LEV followed by a small TEV is a typical flow structure observed for the prescribed effective angle of attack [26]. A strong concentration of vortices are observed during the initial formation of $\mathrm{LEV}, t / T=0.083$ to 0.333 , but it rapidly decays as the size of the LEV is increased. From $t / T=0.250$ to 0.583 , a trail of small vortical structures with similar size and spacing are observed with the starting location at the trailing edge of the flat plate. These structures are created as a part of shear layer induced at the trailing edge of the flat plate as it performs plunging and rotation maneuver, and they are also captured in the flow visualization.

The force measurements for case 1 are shown in Figure 8. All the values are normalized as discussed in the 'Methodology' section. The tangential $\left(F_{x}\right)$ and the normal $\left(F_{y}\right)$ forces are normalized using the same normalization factor for the lift and drag coefficients. The small dotted lines above and below the dark line represent $95 \%$ confidence interval for the 500 phase-averaged measurements in the tangential and normal force plots. The confidence interval is approximately \pm 0.02 in normalized scale and it is only visible in tangential force plot due to the resolution of the y-axis. Small confidence interval over 500 samples indicates that the force measurements were reproducible with a maximum error of approximately \pm 0.02 in the lift and drag coefficients. The tangential force created by the case 1 kinematic is an order of magnitude small compared to the normal force generation. This evidently results in strong resemblance between the normal force and the lift coefficient time history. The lift coefficient time history exhibits a sinusoidal profile that is correlated to the effective angle of attack profile. The peak lift coefficient exceeds a value of 2 with a mean value of 0.362 . The drag coefficient time history shows two thrust peaks at $t / T=0.25$ and 0.75 . At $t / T=0.25$, it produces high thrust and lift coefficient values whereas high thrust and down force is recorded at $t / T=0.75$. From the PIV vorticity contours, the LEV resides on top of the 
flat plate at $t / T=0.50$ and it records similar to the mean lift coefficient value and highest drag coefficient. The mean drag coefficient was computed to be -0.137 which indicated that case 1 is a thrust producing maneuver.

The mean power coefficient value of 0.39 was used to compute the thrust efficiency of case 1 maneuver, and the resulting thrust efficiency was approximately $35 \%$. The power coefficient exhibits dual peaks similar to the drag coefficient profile. The peaks at $t / T=0.25$ and 0.75 are expected since the plunging motion reaches its maximum velocity at these points along with maximum and minimum values of lift coefficients. The moment coefficient shows different trend compared to the lift and drag coefficients; a sharp peak at $t / T \approx 0.4$ record a strong positive moment coefficient with mean of -0.07 . From the sign convention described in the 'Methodology' section, a negative moment coefficient indicates that the motion produces overall pitching up moment about the quarter-chord location.
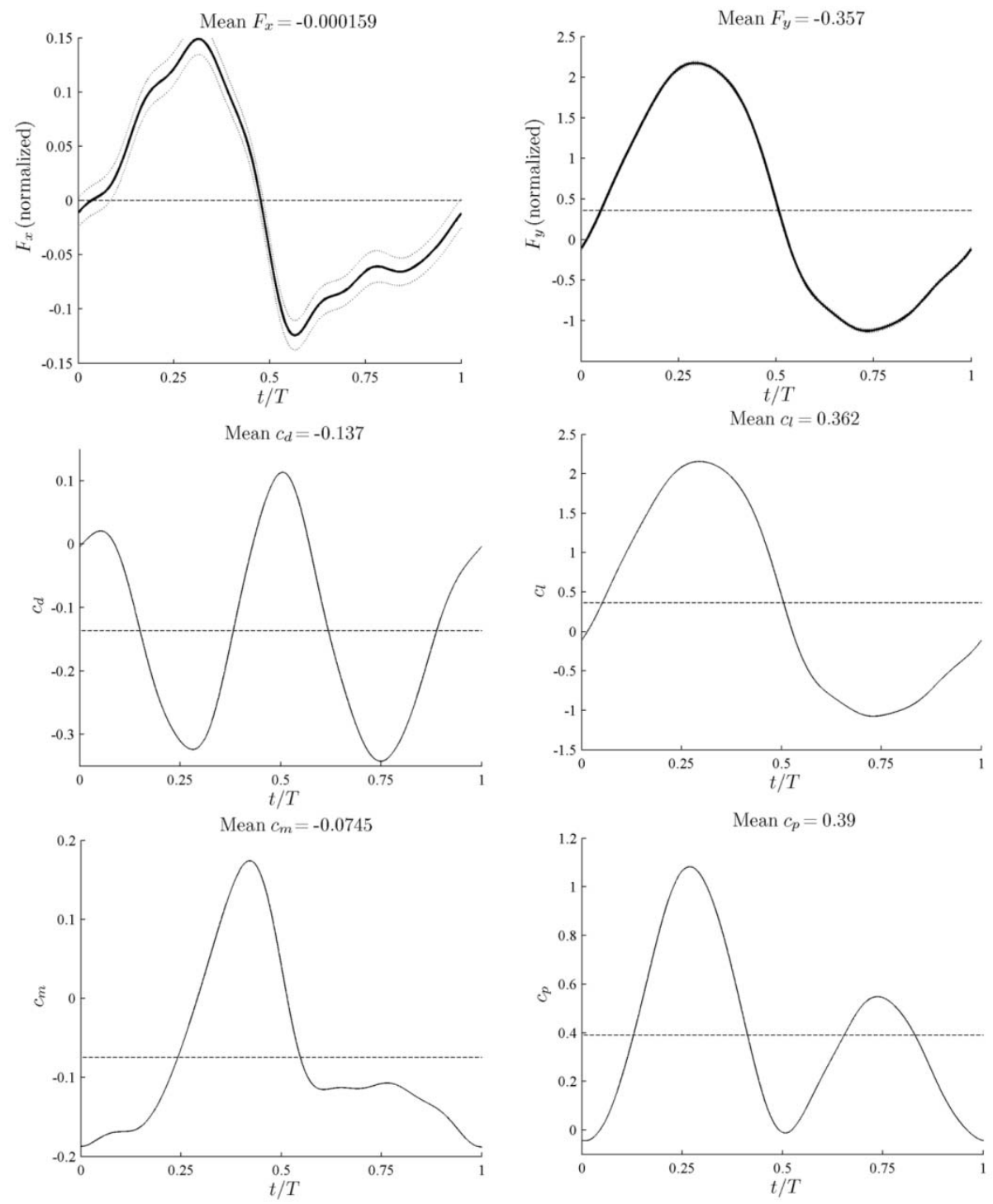

Figure 8. Case 1: Summarized force measurement results showing tangential force $\left(F_{x}\right)$, normal force $\left(F_{y}\right)$, drag coefficient $\left(c_{d}\right)$, lift coefficient $\left(c_{l}\right)$, moment coefficient $\left(c_{m}\right)$, and power coefficient $\left(c_{p}\right)$. 


\section{Case $2(k=1.0, S t=0.32)$}

The same experimental techniques from case 1 were applied to case 2 kinematic and the summarized flow visualization and PIV measurements are shown in Figure A3 for the down stroke motion and Figure A4 for the up stroke motion. Again, the flow topology resulting from case 2 kinematic is similar to past experiments conducted at different $k$-St combination and $R e$ for a flat plate, however, there are differences that are worth noting. The development of LEV is delayed compared to case 1 which is expected with increased reduced frequency; the sign of LEV development does not show until $t / T=0.250$. The size of the LEV is smaller compared to that of case 1 as the faster motion of case 2 does not allow the LEV to grow in size. The up stroke motion is in effect when the LEV is convected approximately half-chord downstream from the leading edge of the flat plate and the concentrated vorticity values in the LEV from $t / T=0.50$ to 0.75 suggests that the up stroke motion prohibits the LEV to grow in size; the up stroke motion stops the shear layer that feeds the LEV and this is shown at $t / T=0.583$. From $t / T=0.583$ onwards, small vortical structures are observed with concentrated vorticity from the leading edge of the flat plate. These small vortical structures were also observed in Baik et al. [32] during up stroke motion for a flat plate with same effective angle of attack at $R e=10,000$. The LEV detaches from the flat plate at $t / T=0.750$ and a small TEV is observed between the LEV and the trailing edge of the flat plate at $t / T=$ 0.833. The geometric angle of attack of the flat plate at $t / T=0.75$ is $41.7^{\circ}$ and the detached LEV is convected downstream with a significant downward motion induced by the high geometric angle of attack. The detached LEV can be observed from $t / T=0.000$ to 0.417 and the size of the LEV does not change while the less concentrated vorticity at the core indicates viscous dissipation. Similar to case 1, a trail of small vortical structures at the trailing edge of the flat plate is observed from $t / T=0.333$ to 0.667 . The size and the spacing between these structures are comparable to case 1 but higher vorticity concentration is recorded for case 2. This is a strong indication that the formation of these structures are due to the instability of the shear layer created at the trailing edge while the difference in concentration is a result of faster motion kinematic.

The force measurements for case 2 kinematic are shown in Figure 9. The main highlight for case 2 kinematic is the measured normal force component; the magnitude reaches up to 4.5 with a dual peak profile that is different from a sinusoidal profile observed for case 1 . The tangential force does show different profile from case 1 but the magnitude remains similar for both cases which indicate that the contribution from tangential force is negligible compared to the normal force contribution. In addition, similar 95\% confidence interval as case 1 was measured for case 2. After projecting the tangential and normal forces to compute lift and drag coefficients, the drag coefficient profile shows a sinusoidal behavior and the lift coefficient profile resembles the normal component despite the fact that there is a large variation in the geometric angle of attack. A thrust peak with a value of approximately 2.1 is measured at $t / T=0.25$ and a drag peak with a value of approximately 1.3 is measured at $t / T=0.75$. This is contrary to the dual thrust peak observed for case 1 at these phases. It is evident that a significant part of the normal force was projected towards thrust and drag due to the high magnitude of geometric angle of attack at these phases. The geometric angle of attack at $t / T=0.25$ and 0.50 are $-25.7^{\circ}$ and $41.7^{\circ}$, respectively. The mean drag coefficient value of -0.284 indicates a thrust producing maneuver and this value is twice the value of mean thrust coefficient for case 1 . The lift coefficient shows two peaks at $t / T \approx$ 0.25 and 0.50 with a value of approximately 4 and 3.5, respectively. There exists a sudden decrease in lift between $t / T=0.50$ and 0.75 and this coincides with the start of the up stroke motion. It is interesting to note that the LEV resides on the suction side of the flat plate as the model performs the up stroke motion. 
It is clear that inertial force created by the up stroke motion overwhelms the lift force generated by the attached LEV, causing a drop in the lift coefficient.

The mean power coefficient was computed to be 0.686 and the resulting thrust efficiency for case 2 was approximately $41 \%$. A high value of power coefficient is recorded during the down stroke of the motion, especially at $t / T=0.25$ which recorded a peak value that is associated with the highest thrust coefficient production. The large consumption of power during the down stroke was offset during the up stroke motion where the presence of lift reduced the overall power consumption between $t / T=0.50$ and 0.75. The moment coefficient profile displays similarities to the lift coefficient time history with a peak located at $t / T=0.50$. A large value of positive moment indicates that a strong pitching down moment is present at the quarter-chord location at $t / T=0.50$.
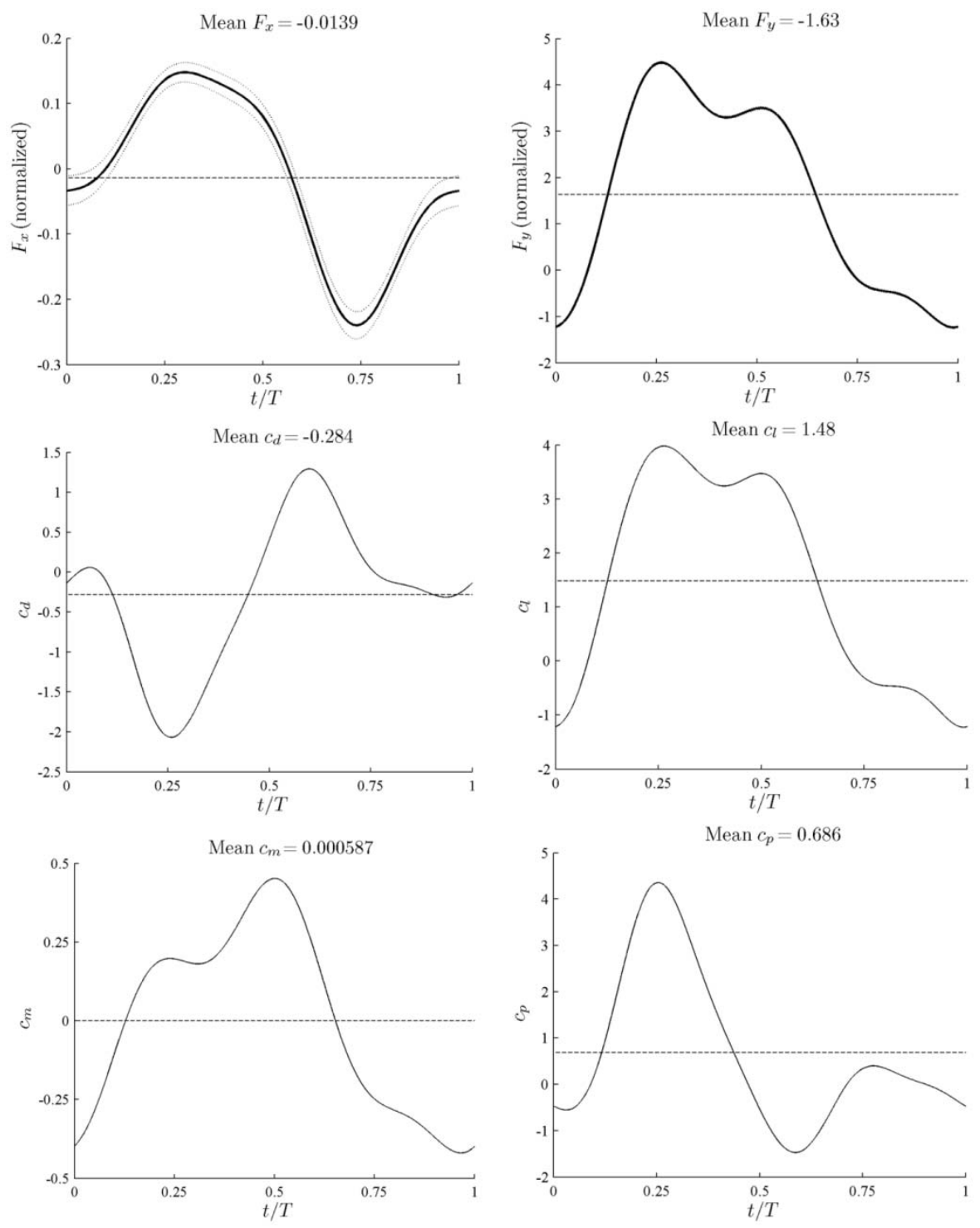

Figure 9. Case 2: Summarized force measurement results showing tangential force $\left(F_{x}\right)$, normal force $\left(F_{y}\right)$, drag coefficient $\left(c_{d}\right)$, lift coefficient $\left(c_{l}\right)$, moment coefficient $\left(c_{m}\right)$, and power coefficient $\left(c_{p}\right)$. 

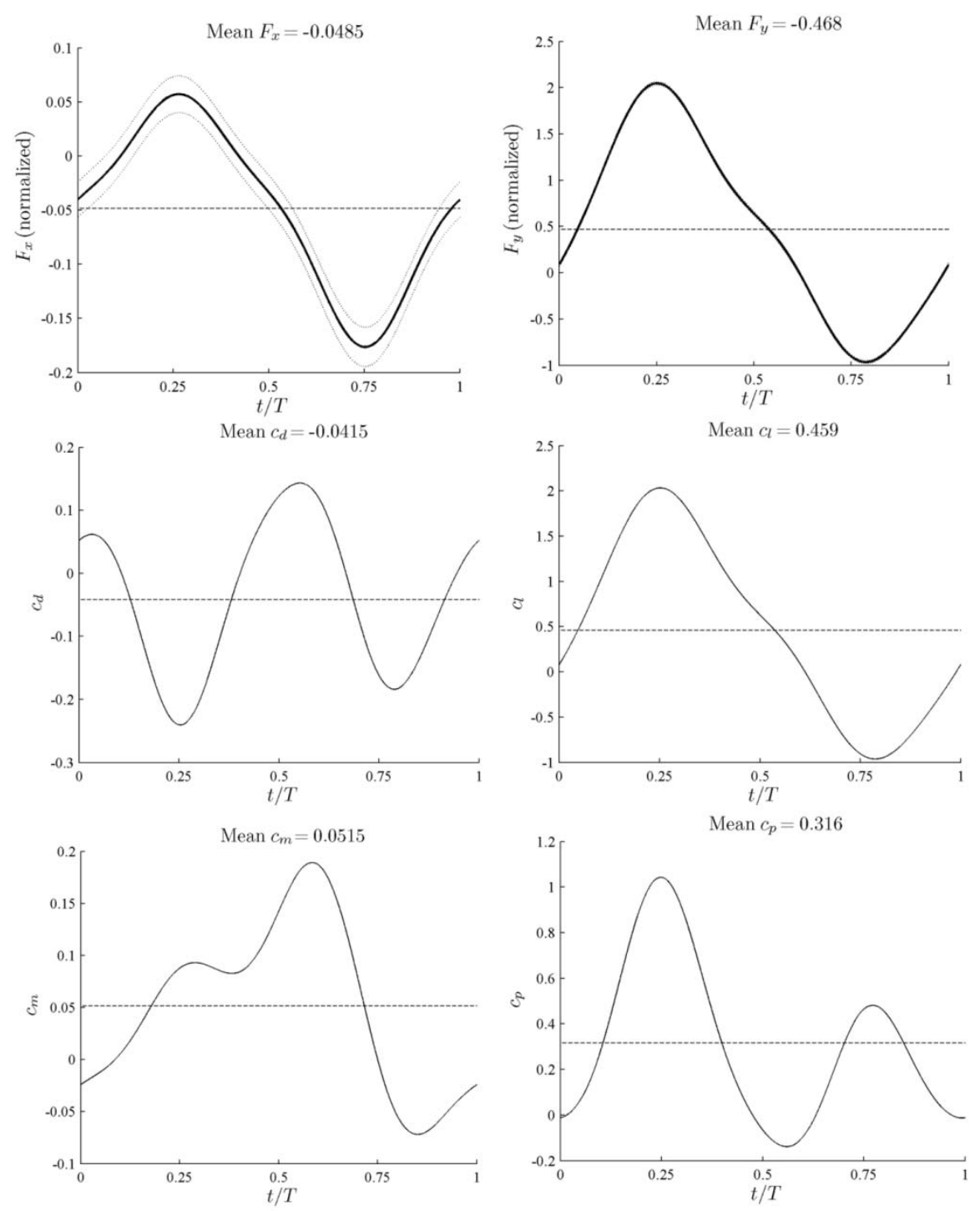

Figure 10. Case 3: Summarized force measurement results showing tangential force $\left(F_{x}\right)$, normal force $\left(F_{y}\right)$, drag coefficient $\left(c_{d}\right)$, lift coefficient $\left(c_{l}\right)$, moment coefficient $\left(c_{m}\right)$, and power coefficient $\left(c_{p}\right)$.

D. Case $3(k=1.0, S t=0.16)$

Case 3 serves as an intermediate case that links cases 1 and 2 together; the $S t=0.16$ is the same with case 1 and the $k=1.0$ is the same with case 2 . The summarized flow visualization and PIV measurements are shown in Figure A5 for the down stroke motion and Figure A6 for the up stroke motion. The flow topology shows strong similarities with case 2; the delayed LEV formation time, LEV size, peak vorticity values and their locations, and LEV detachment location and the formation of TEV is in good agreement with case 2. This is not surprising given they share the same $k$ value of 1.0 which seem to govern the formation time of LEV for this particular effective angle of attack profile and therefore allowing the flow structure to be similar around the pitching and plunging flat plate. As the LEV starts to develop at $t / T=$ 0.25 , the amount of concentrated vorticity within the LEV is larger compared to case 2 . The size and the strength of the LEV involving computation of LEV circulation will be discussed in more detail in the latter part of the paper. The LEV detachment occurs at $t / T=0.833$ and a small TEV is observed between 
the LEV and the trailing edge of the flat plate. The difference between cases 2 and 3 at this phase is the trajectory of the LEV after detachment; the geometric angle of attack for case 3 at $t / T=0.833$ is not as steep as case 2 and therefore the detached LEV convects downstream without much down ward velocity, shown from $t / T=0.000$ to 0.417 . A trail of small vortical structure at the trailing edge of the flat plate have similar vorticity strength as case 2 , confirming that the strength of these vortical structures and the motion frequency are positively correlated.

The force measurements for case 3 are summarized in Figure 10. Contrary to the similarities found in the flow topology between cases 2 and 3, it is rather surprising to observe that the force measurements for case 3 resembles to the case 1 measurements. A good level of agreement is present at qualitative and quantitative level. The sinusoidal profile of tangential and normal forces is observed with similar 95\% confidence interval levels. A dual thrust peaks are present at $t / T \approx 0.25$ and 0.75 , but the mean value of thrust coefficient of 0.0415 is approximately 3 times less than case 1 . However, there is an improvement in the mean lift coefficient which recorded 0.459 . Compared to 0.362 of case 1 , it is $27 \%$ increase in lift. The lift coefficient profile indicates that the residence time at high magnitude lift coefficient is small; the lift peak is achieve at $t / T=0.25$ but it rapidly decreases at subsequent phase, and similar trend is observed at $t / T \approx 0.80$.

The moment coefficient profile is the only profile that shows discrepancy with case 1 but similarity with case 2 . This is an indicator that the moment coefficient profile is correlated to the pitching frequency since $k$ value is the same for cases 2 and 3. The mean moment coefficient is 0.0515 which indicates an overall pitching down moment for the maneuver. The thrust efficiency suffered from low mean thrust coefficient while the mean power coefficient remained approximately the same between cases 1 and 3 . The computed thrust efficiency was $13.2 \%$ which is approximately $1 / 3$ of the thrust efficiency of case 1 . The power consumption peaks at $t / T=0.25$ and 0.75 are primarily due to the plunging motion counteracting with the lift force.

\section{E. Flux equation}

The main idea behind FE analysis is not to verify the accuracy of the force sensors, but to gain insight on the components of the force by analyzing different components contributing to the overall force acting on the flat plate; this is the strength of using FE to compute the force coefficients which direct force measurements will not be able to provide. The comparison of lift and drag coefficients computed from FE and force measurements for all cases is shown in Figure 11. The qualitative agreement between the two methods is established although the quantitative agreement could be better. In terms of capturing the trend in the time history, the lift coefficient profile seems to perform better than the drag coefficient profiles; FE is constantly over- or under-predicting the drag coefficient values compared to the force measurements. For case 2, the drag coefficient values from the FE does not record high peaks and the first peak seem to occur with a phase lag. The comparison is improved for the lift coefficient although the FE does not capture the first peak in the lift coefficient. The best qualitative comparison is found for case 3 lift coefficients where the entire force measurements seem to have an offset of approximately 0.5 in $c_{l}$. There are several factors that introduce error in the computed lift and drag coefficient values from FE: discontinuity introduced from stitching of the images, integration error due to insufficient spatial and/or time resolution, and 3D flow structures in a 2D flow setup. To resolve these issues, additional experimental works are required to verify the sensitivity of FE on PIV data.

The FE was decomposed into 5 components, namely A, B, C, D, and E, and it is shown in Eq. (13). The overall force, or the 'Total' force, is the sum of all the force components. The main distinction in these components is the terms involved; $\mathrm{C}$ component has information about vorticity, $\mathrm{D}$ component 
possess time rate of change of velocity field, and E component computes the viscous effects on the overall force. Notice that components $\mathrm{C}, \mathrm{D}$, and $\mathrm{E}$ have the origin location as a variable. The breakdown of each force component and the 'Total' force for lift and drag coefficients are summarized in Figure 12. The E component was omitted from the plots because the values were two orders of magnitude smaller than the rest of the terms. The A and B components were added together for more efficient comparison.

$$
\begin{gathered}
\vec{F}=\oint_{S(t)} \vec{n} \cdot \gamma_{\mathrm{flux}} d S, \gamma_{\mathrm{flux}}=A+B+C+D+E \\
A=\frac{1}{2} u^{2} \overline{\bar{I}}, B=-\vec{u} \vec{u}, C=\vec{\omega}(\vec{x} \times \vec{u})-\vec{u}(\vec{x} \times \vec{\omega}), \\
D=-\left[\left(\vec{x} \cdot \frac{d \vec{u}}{d t}\right) \overline{\bar{I}}-\vec{x} \frac{d \vec{u}}{d t}+\frac{d \vec{u}}{d t} \vec{x}\right], E=[\vec{x} \cdot(\nabla \cdot \overline{\bar{T}}) \overline{\bar{I}}-\vec{x}(\nabla \cdot \overline{\bar{T}})]+\overline{\bar{T}}
\end{gathered}
$$

The most dominant term in the FE is the $\mathrm{D}$ component which is the time rate of change of velocity at the control surfaces. This is observed by determining the effect that D component has on the 'Total' force, and the simplest way to determine this is to compare the gradient of both profiles. For most of the lift and drag coefficient profiles, it is observed that the gradient of the D component is in a good agreement with the overall force coefficient time history; the trend observed in the D component influences the 'Total' force effectively. It is difficult to distinguish whether LEV has a direct impact on the D component since the $\mathrm{D}$ component does not involve vorticity as a variable but it does introduce time rate of change of velocity field as the LEV size grows. By considering the effect a vortex has on neighboring fluid particle to a moving flat plate, the moving flat plate will affect the velocity field at a faster rate than a vortex simply due to the fact that the motion occurs at a faster rate than the development of a large vortical structure. This would imply that the D component is more likely to be a contribution from the flat plate motion itself rather than from the generation of a vortex in the flow, and it may explain why case 2 generates more forces than cases 1 and 3.

There are instances where the $\mathrm{C}$ component will compete for the dominance and a good example is shown for case 2 at $t / T=0.167$ to 0.417 where the $\mathrm{C}$ and $\mathrm{D}$ components are offsetting each other to result in a lift coefficient value. There are also instances where the $\mathrm{C}$ component is the dominant contributor to the force. For example, case $1 \mathrm{drag}$ coefficient profile at $t / T=0.00$ to 0.25 shows the $\mathrm{C}$ component affecting the overall drag coefficient, and case 3 lift coefficient profile at $t / T=0.667$ to 0.917 also exhibits similar features where the $\mathrm{D}$ component force is offset by the $\mathrm{A}+\mathrm{B}$ component forces. The $\mathrm{C}$ component force involves vorticity and it will be further discussed in the vortex dynamics section.

The identification of a dominant force component not only distinguishes which terms are important to the overall force generation, it also suggests the types of data required for an accurate force coefficient computation. Since the D component is a dominant component for the most part, it is a necessary requirement that the time derivatives of the velocity vector field at the control surfaces are computed accurately. If the $\mathrm{C}$ component is a dominant component, then the vorticity should be sufficiently converged. Acquiring accurate data set will also help in resolving problems associated with origins and control volume dependence on the force coefficient computation. 
Case 1

○ FE — Force Measurement
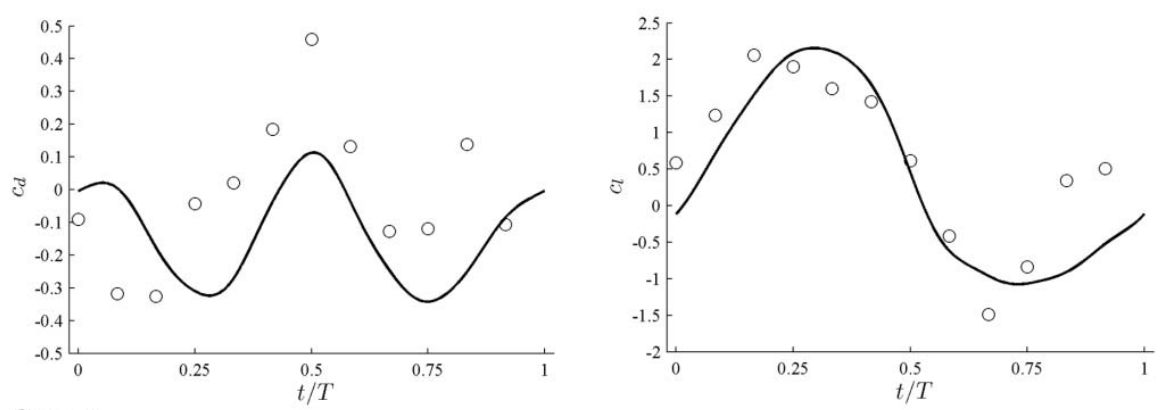

Case 2

○ $\mathrm{FE} \longrightarrow$ Force Measurement
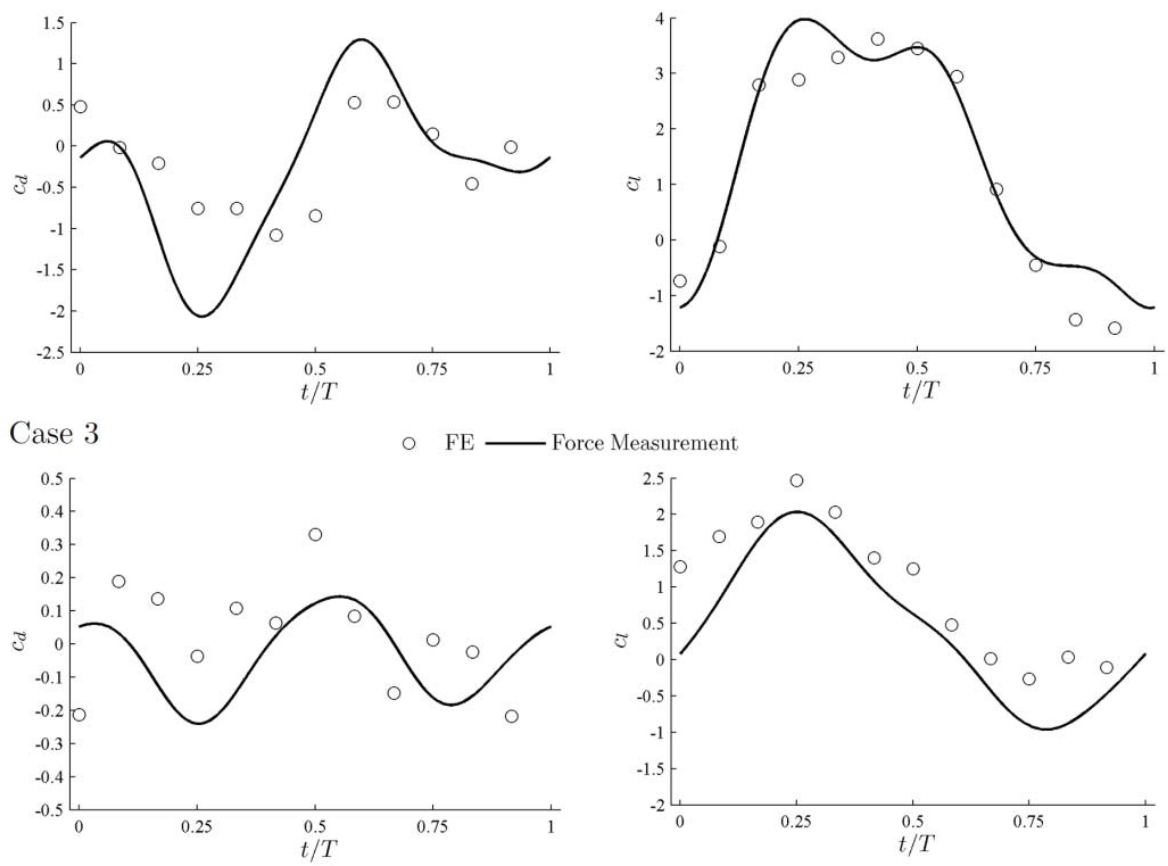

Figure 11. Comparison of lift and drag coefficients computed from FE and force measurements for all cases.

\section{F. Effects of inertia and vortex dynamics}

The force measurements for all 3 cases are compiled and plotted against each other for comparison as shown in Figure 13. Cases 1 and 3 display strong similarities in the normal force coefficient despite having different flow topology arising from different $k$ value. The tangential force coefficients do not show any specific trends between all 3 cases, but the values are small compared to the lift coefficients. It is clear that case 2 outperforms cases 1 and 3 in both thrust and lift generation; the recorded thrust peak is an order of magnitude higher, and the recorded lift peak is more than double compared to other 2 cases. Case 1 shows more rapid decrease in lift from $t / T=0.30$ to 0.75 compared to case 3 , and this difference is examined in detail by considering the force differences between 2 cases. There are 2 comparisons done in total: cases 1 and 3, and cases 2 and 3. Cases 1 and 3 share the same $S t$ but different $k$ values and the force coefficients are in a good agreement with each other. Cases 2 and 3 share the same $k$ but different $S t$ values and they share similar flow topology. The two comparisons are shown in Figure 14. 


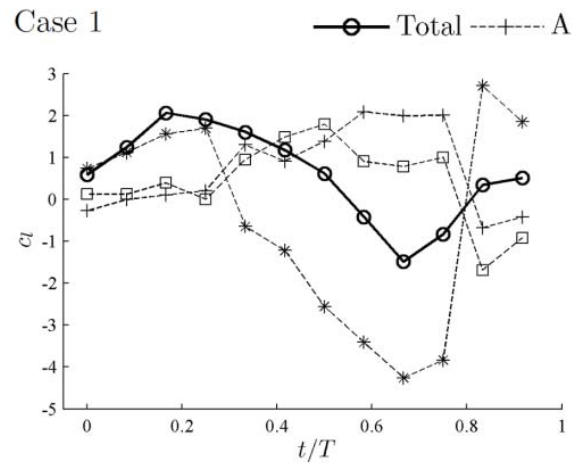

$\mathrm{A}+\mathrm{B}---\mathrm{-}^{--\cdots} \mathrm{C}-\cdots *-\cdots \mathrm{D}$

Case 2
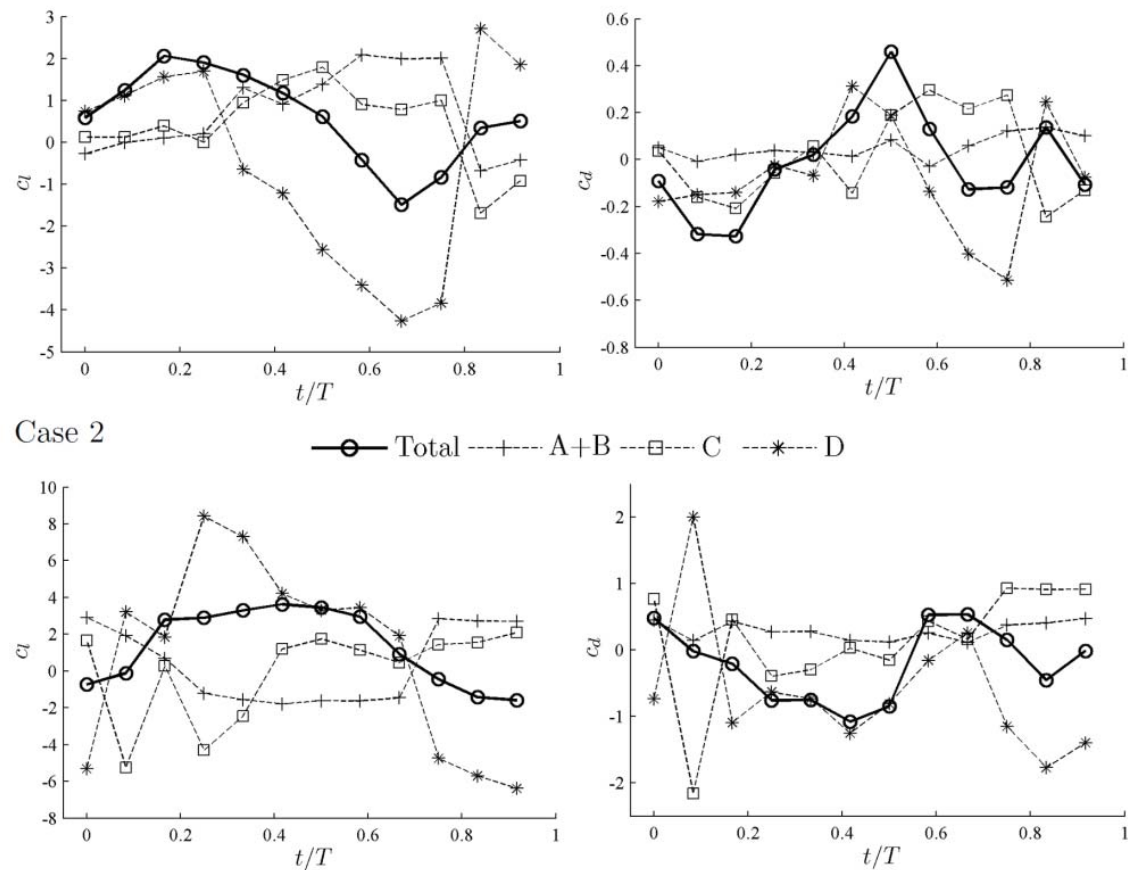

$\mathrm{A}+\mathrm{B}---\boxminus-\cdots \mathrm{C}-\cdots+\cdots$

Case 3
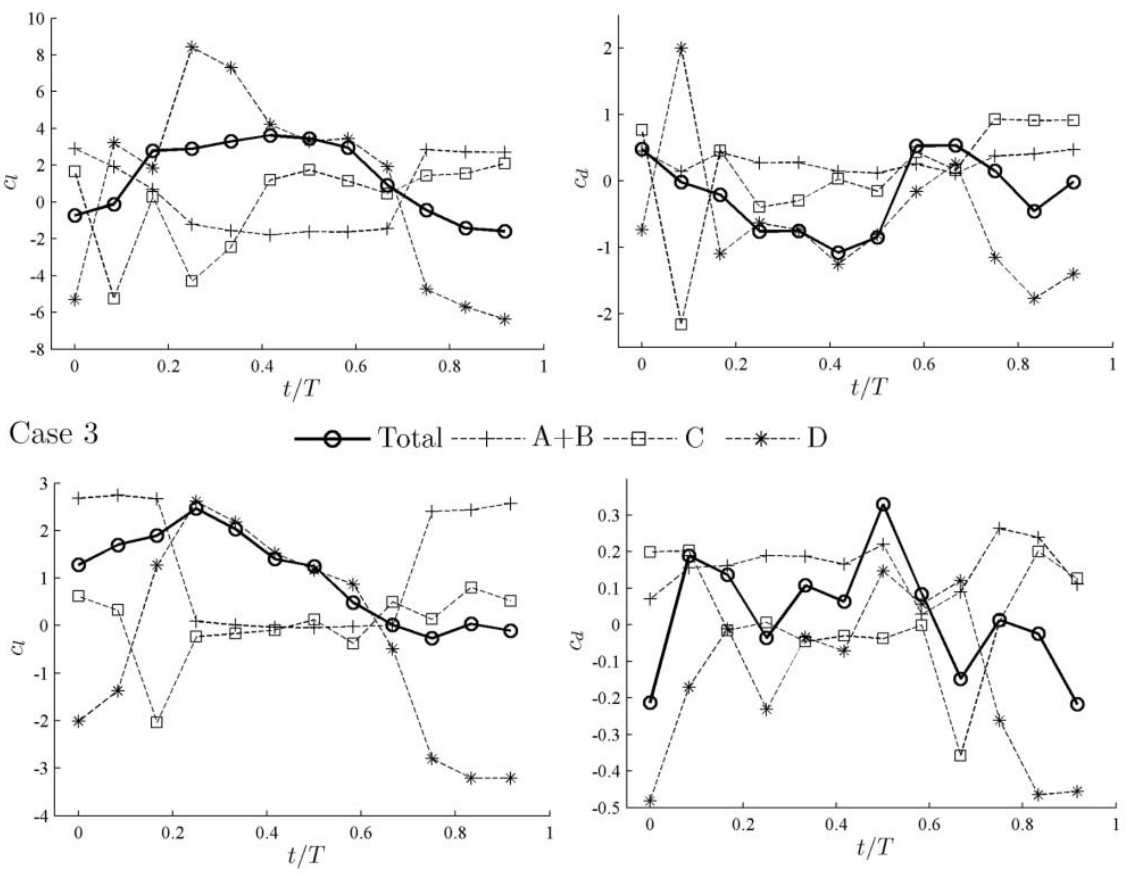

Figure 12. Breakdown of components in FE contributing to the overall force; See Eq. (13) for terms in A, B, C, and D.

The main highlight from Figure 14 is the effect of inertia and vortex dynamics that have on the discrepancy in force generation between cases. The force components from FE lift coefficients were analyzed to distinguish the dominant contributor to the overall force and it is shown in Figure 15. The drag coefficients comparison was not performed on FE due to large amount of fluctuations present relative to the magnitude of the drag force, and therefore a lack of qualitative consistency. For cases 1 and 3, it is evident from Figure 15 that the $\mathrm{C}$ component is the dominant contributor to the overall lift force; the combined components of $\mathrm{A}$ and $\mathrm{B}$ offsets the $\mathrm{D}$ component, and the trend between the overall drag coefficient and the $\mathrm{C}$ component is in a good agreement. The $\mathrm{C}$ component is associated with the vorticity in the flow field and this implies that the discrepancy in the force generation between cases 1 and 3 is mainly due to the circulatory effects, not inertial effects. Given that the plunging motion is proportional to $S t$, comparable inertial effects between the two cases seem plausible since they share the same $S t=0.16$. In order to assess the cause of lift coefficient discrepancy in more detail, the LEV size and strength calculation was performed and it is shown in Figure 16. The strength of the circulation is measured by the 
normalized magnitude of the circulation $\left(\left|\Gamma^{*}\right|\right)$ within LEV boundaries. The normalized circulation at $t / T$ $\approx 0.40$ is approximately 1.5 for case 1 and 1.0 for case 3 , and given the fact that both cases have LEV present on top of the flat plate explains why case 1 exhibits higher lift coefficient compared to case 3 . From $t / T=0.50$ to 0.75 , case 3 exhibits more lift than case 1 because the LEV is still attached and on top of the flat plate whereas the LEV detaches for case 1 at the start of the up stroke hence the loss of lift. The vorticity contours for case 1 and 3 are shown in Figure A1 and A5, respectively. From Figure 17(b), both cases show linear growth in the strength of LEV with case 1 growing at a faster rate. Both cases reaches maximum LEV strength at $t / T=0.50$ where a decrease in normalization circulation value is reported at a subsequent phase. The size of LEV is larger for case 1 and the rate of LEV size growth is nonlinear whereas the case 3 shows almost a linear growth in LEV size.
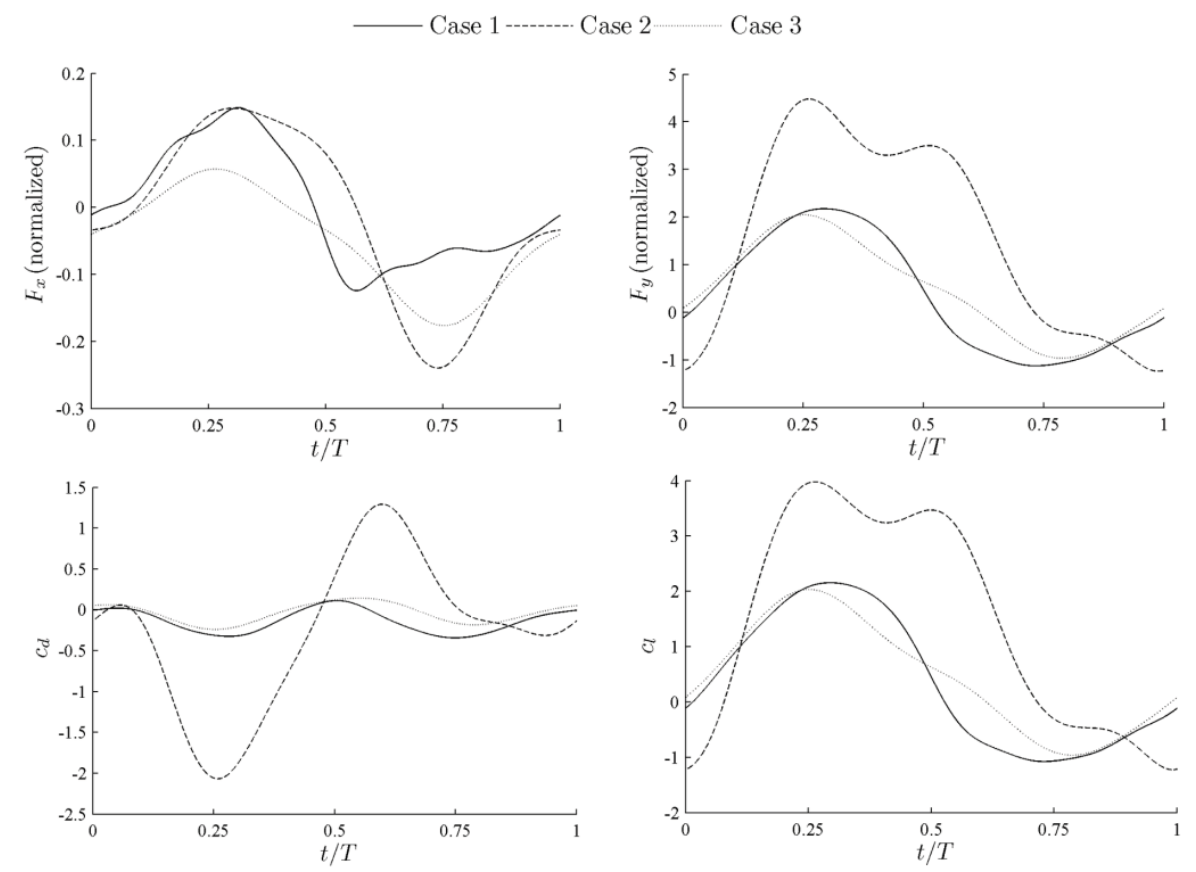

Figure 13. Comparison of force sensor measurements for cases 1, 2 and 3.

For cases 2 and 3, the main difference in the force generation is caused by the inertial effect; the $S t$ is doubled for case 2 compared to case 3. It is not plausible to account for the large discrepancy in force coefficients solely due to circulatory effect, especially when the flow topology for both cases is similar. It is evident from Figure 15 that the $\mathrm{C}$ and $\mathrm{D}$ components are competing for the overall lift contribution for cases 2 and 3. A rapid increase in lift coefficient from $t / T=0.00$ to 0.25 is due to inertial acceleration at the start of the motion. Between $t / T=0.417$ and 0.583 is where the D component approximately equals to the difference in lift generation for two cases, and this is a strong evidence that the non-circulatory effects are the source of additional lift. The decrease in lift after $t / T=0.583$ also follows the trend of the D component. The strength of the LEV for case 2 was computed to be slightly higher than case 3 with similar qualitative feature such as peak strength at $t / T=0.50$. There are two factors that contribute to the discrepancy in the LEV strength: the pitching rate of the flat plate and the development characteristic of the LEV. The difference in the pitching rate definitely introduces extra circulation in the control volume and the subtle differences in the LEV strength found in Baik et al. [25] was believed to be caused by the 
difference in pitching rate for constant $k$ kinematics. Another explanation is the fact that the area of LEV for case 2 was measured larger than the LEV observed in case 3. This observation was not available in previous study primarily due to the fact that the $k$ value was not high enough. The LEV for case 2 starts to develop during the end of the up stroke motion rather than $t / T=0.00$. In Figure A4, a small rotation dominated zone is captured at the leading edge of the flat plate at $t / T=0.917$ that grows in size to become the LEV in subsequent phases. This effectively shifts the LEV size growth curve to a new origin to $t / T \approx$ 0.9 and the resulting curve in Figure 16(b). If the strength of LEV is dominated by the $k$ value, it is a possible that the increase in LEV area will cause higher circulation within the LEV. Further investigation at same $k$ but different $S t$ needs to be performed to verify the effect of $S t$ on the strength of LEV.
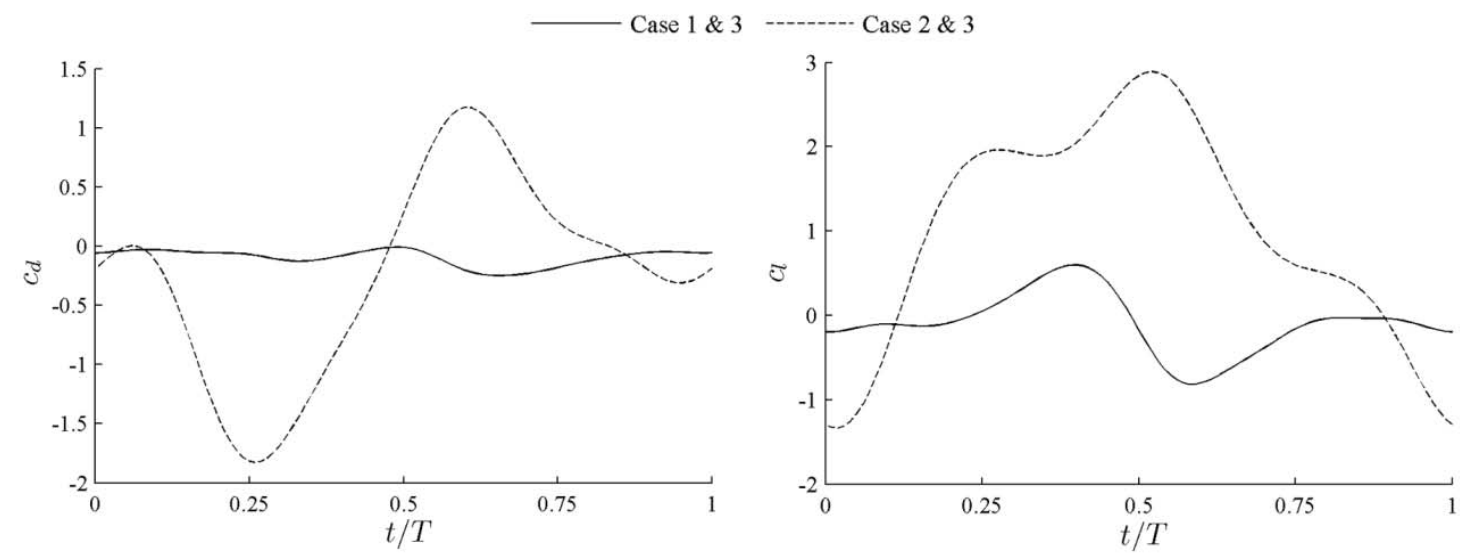

Figure 14. Differences in drag and lift coefficients between cases 1 and 3, and cases 2 and 3. ('Case 1\&3' denotes force coefficients from case 1 subtracted by case 3)
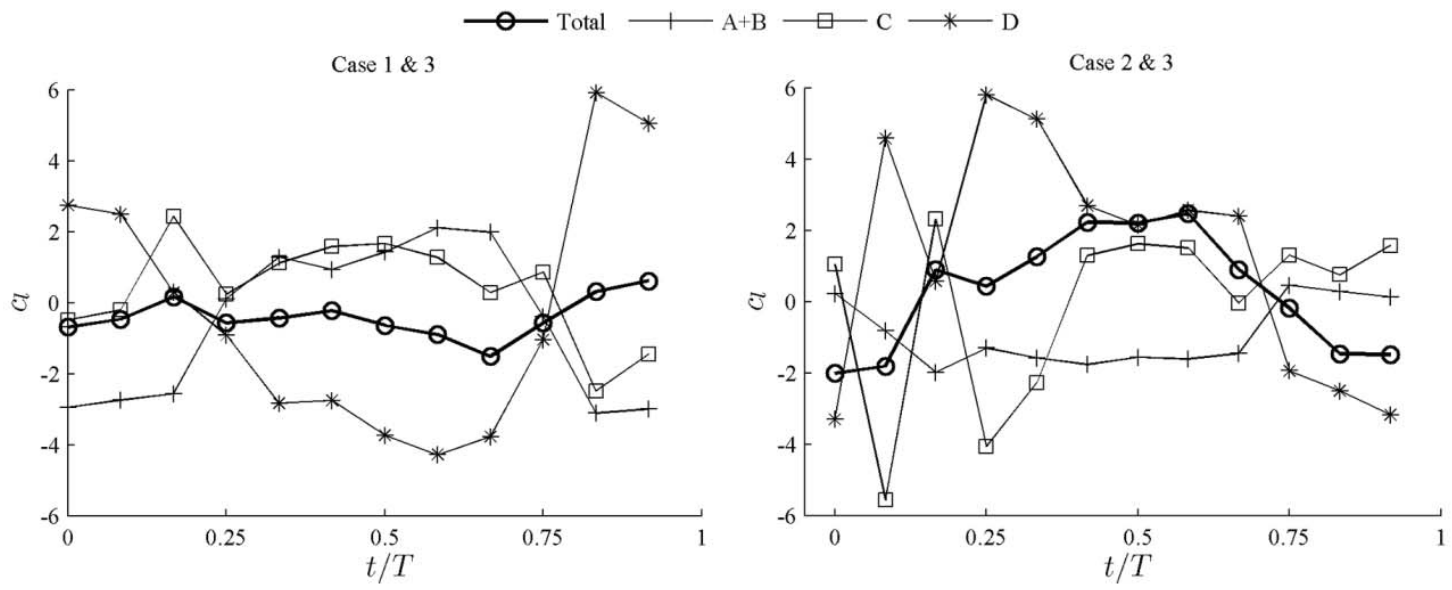

Figure 15. Breakdown of force components in FE for lift coefficients between cases 1 and 3, and cases 2 and 3. ('Case 1\&3' denotes force coefficients from case 1 subtracted by case 3 )

The LEV core trajectory exists independent of the geometric angle of attack of the flat plate as long as the effective angle of attack of the prescribed motion remains constant. In order to verify the repeatability of previous findings, the LEV core locations were plotted on top of the previous cases, and it is shown in Figure 17. The conclusion from the LEV core trajectory analysis is that the trajectory 
becomes distorted at high $S t$ kinematics, and not high $k$. The fit between the recent cases and previous cases are good; two cases from previous study [25] were chosen with $S t=0.15$ because cases 1 and 3 have $S t=0.16$. The $R e$ is 5,000 for the current cases and 10,000 for previous cases. A second order polynomial was curve fitted through the LEV core locations with an exception of case 2 coordinates since they did not fit the trend. A good fit implies that the LEV core trajectory is repeatable and the Re has a negligible effect on the LEV trajectory. Case 2 LEV core trajectory showed mirror image about the x-axis of the LEV trajectory at lower St kinematics. This may be associated with the high magnitude geometric angle of attack during the motion accompanied by the high pitching rates.

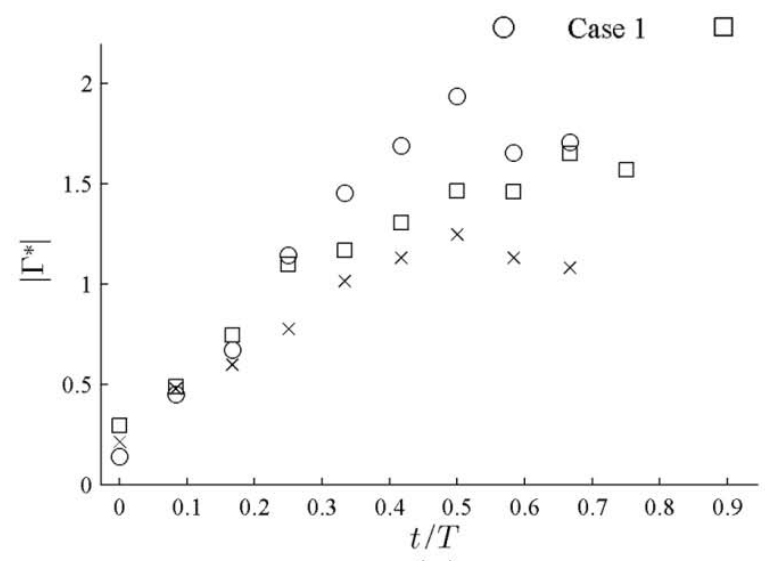

(a)

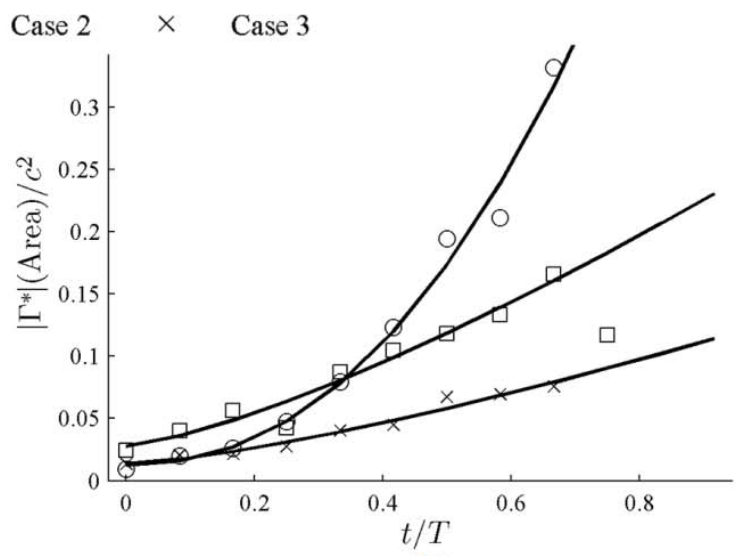

(b)

Figure 16. (a) Normalized circulation of LE, and (b) normalized LEV area for all cases.

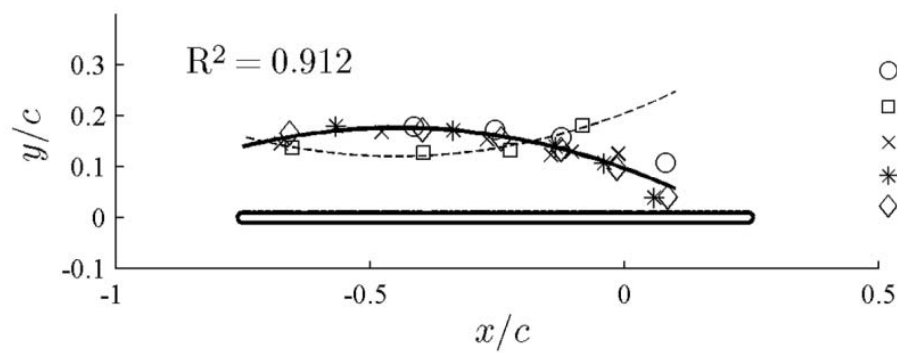

○ Case $1(S t=0.16, k=0.5)$

Case $2(S t=0.32, k=1.0)$

Case $3(S t=0.16, k=1.0)$

Baik et al. $(S t=0.15, k=0.471)$

Baik et al. $(S t=0.15, k=0.628)$

Figure 17. Vortex core trajectory with respect to the flat plate for various cases.

\section{Conclusions}

An experimental investigation of pitching and plunging flat plate at a prescribed effective angle of attack in the $S t$ range of 0.16 to 0.32 and $k$ range of 0.5 to 1.0 is presented. Force measurements and PIV technique were used to analyze and quantify the unsteady flow field created by the wing kinematics. A momentum-balance based non-intrusive force measurement technique and a systematic vortex detection algorithm were implemented as a post-processing tool for PIV data to gain insight on the unsteady force generation and vortex dynamics. The main conclusions are the following: 
- The inertial force, which is proportional to the $S t$, is a dominant contributor to the overall force generation in pitching and plunging flat plate. The qualitative accuracy of 'Flux Equation' enabled a detailed analysis of force components associated with the force generation and it was found that the time rate of change of the velocity field contributes to the overall force generation more than vorticity in the flow field. The circulatory component, such as LEV, was unable to account for the differences in the force generated between cases 2 and 3 where flow topology and the computed LEV strength was comparable to each other.

- The circulatory component of the force contributes to the difference in the force generation between kinematics with the same $S t$ by manipulating the development and convection of LEV through reduced frequency. Similar amount of power was consumed for cases 1 and 3 and the computed thrust efficiency was $35 \%$ and $13 \%$, respectively. The difference in drag and lift coefficient was caused by the size and location of the LEV in the flow field with respect to the flat plate. The delayed development of LEV due to higher $k$ value was consistent with the previous findings.

- The normal force component is more significant than the tangential force in force generation. The pitching amplitude increased as the $S t$ increased from conserving the effective angle of attack profile, which led to increase in thrust and drag production through projection of normal force. The recorded propulsive efficiency of case 2 was approximately $42 \%$ with significant amount of thrust resulting from the projection of the normal force to the thrust component.

- The LEV core trajectory with respect to the flat plate holds for kinematic up to $S t=0.20$. The repeatability was established at different $R e$, indicating the insignificance of $R e$ effect on the dynamics of LEV. The trajectory was disrupted for higher $S t=0.32$ kinematic due to high magnitude of geometric angle of attack and higher pitching rates.

- The 'Flux Equation' successfully captured qualitative features of computed force coefficients; the lift coefficients compared better than the drag coefficients due to larger magnitude of force measured in the lift. The condition required to achieve origin and control volume independent solution was tested and successfully implemented. However, sensitivity to the spatial and time resolution of the PIV data need to be further investigated for more quantitatively accurate solutions.

\section{Acknowledgments}

This work has been supported in part by the Air Force Office of Scientific Research's Multidisciplinary University Research Initiative (MURI) and by the Michigan/AFRL (Air Force Research Laboratory)/Boeing Collaborative Center in Aeronautical Sciences. The authors would also like to acknowledge Chris Morrison with the experimental setup. 


\section{Appendix A}

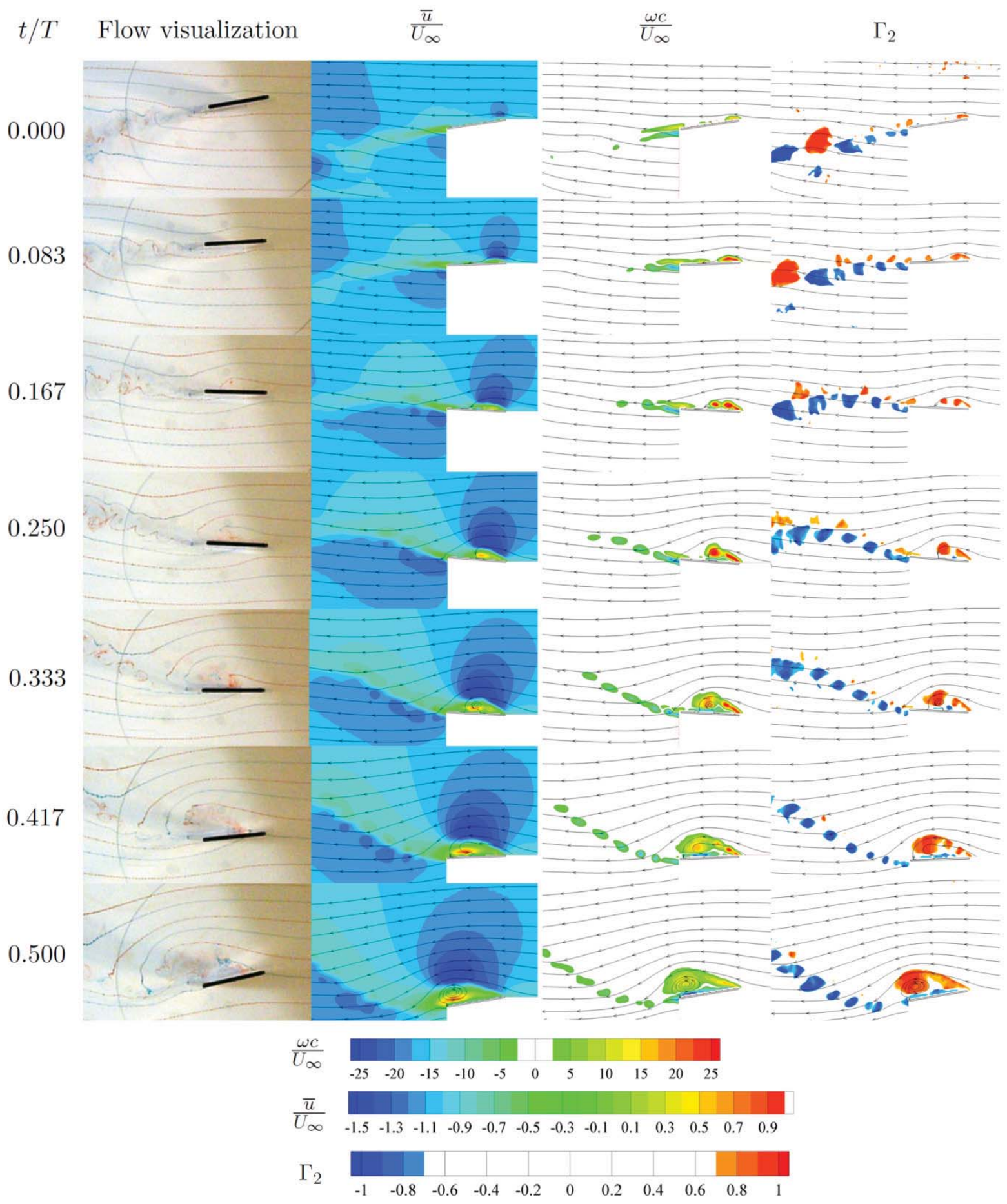

Figure A1. Case 1: Flow visualization, normalized $x$-component velocity contours, normalized vorticity contours at $t / T=$ $0.000,0.083,0.167,0.250,0.333,0.417$, and 0.500 . 


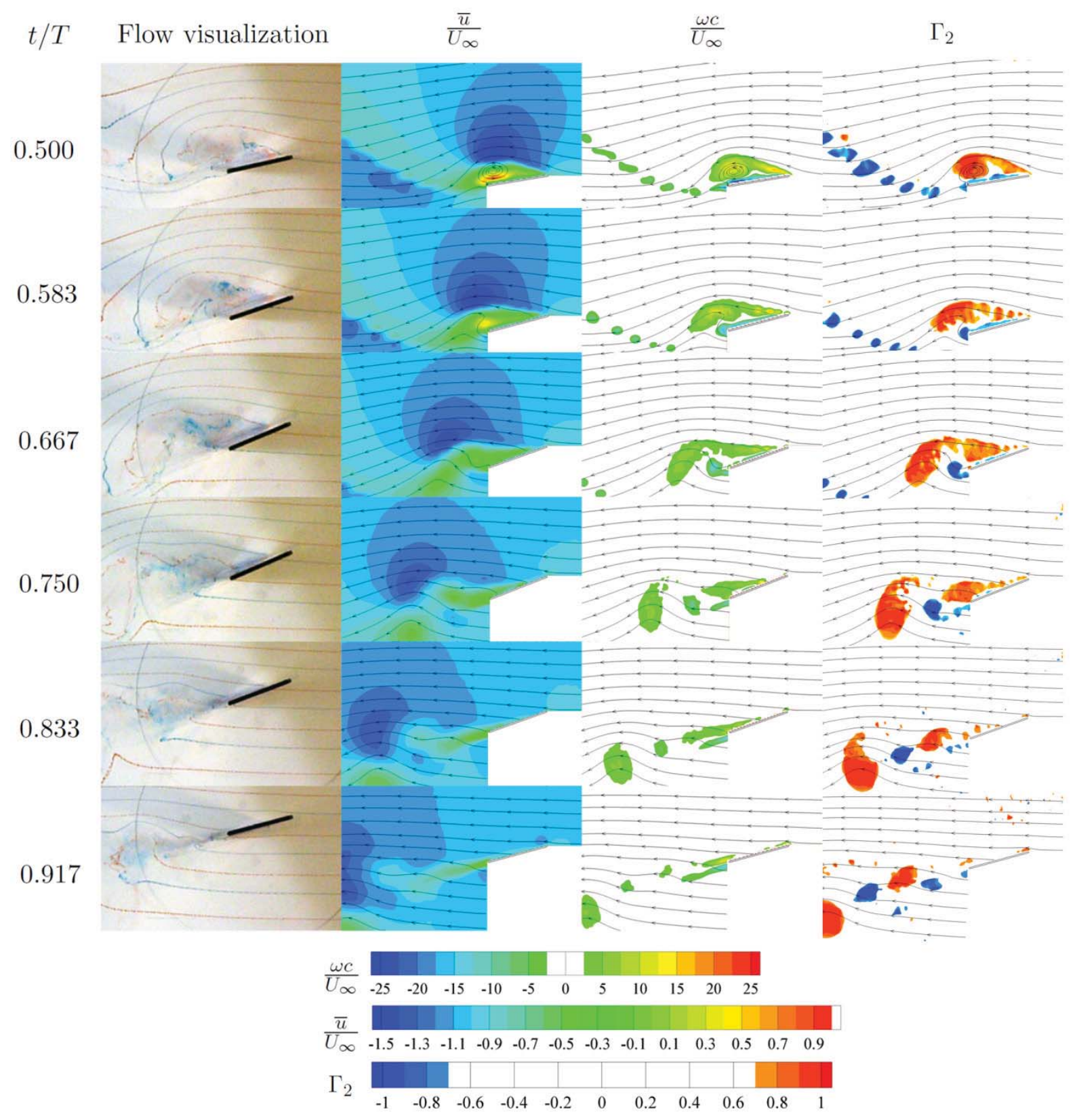

Figure A2. Case 1: Flow visualization, normalized $x$-component velocity contours, normalized vorticity contours at $t / T=$ $0.500,0.583,0.667,0.750,0.833$, and 0.917 . 


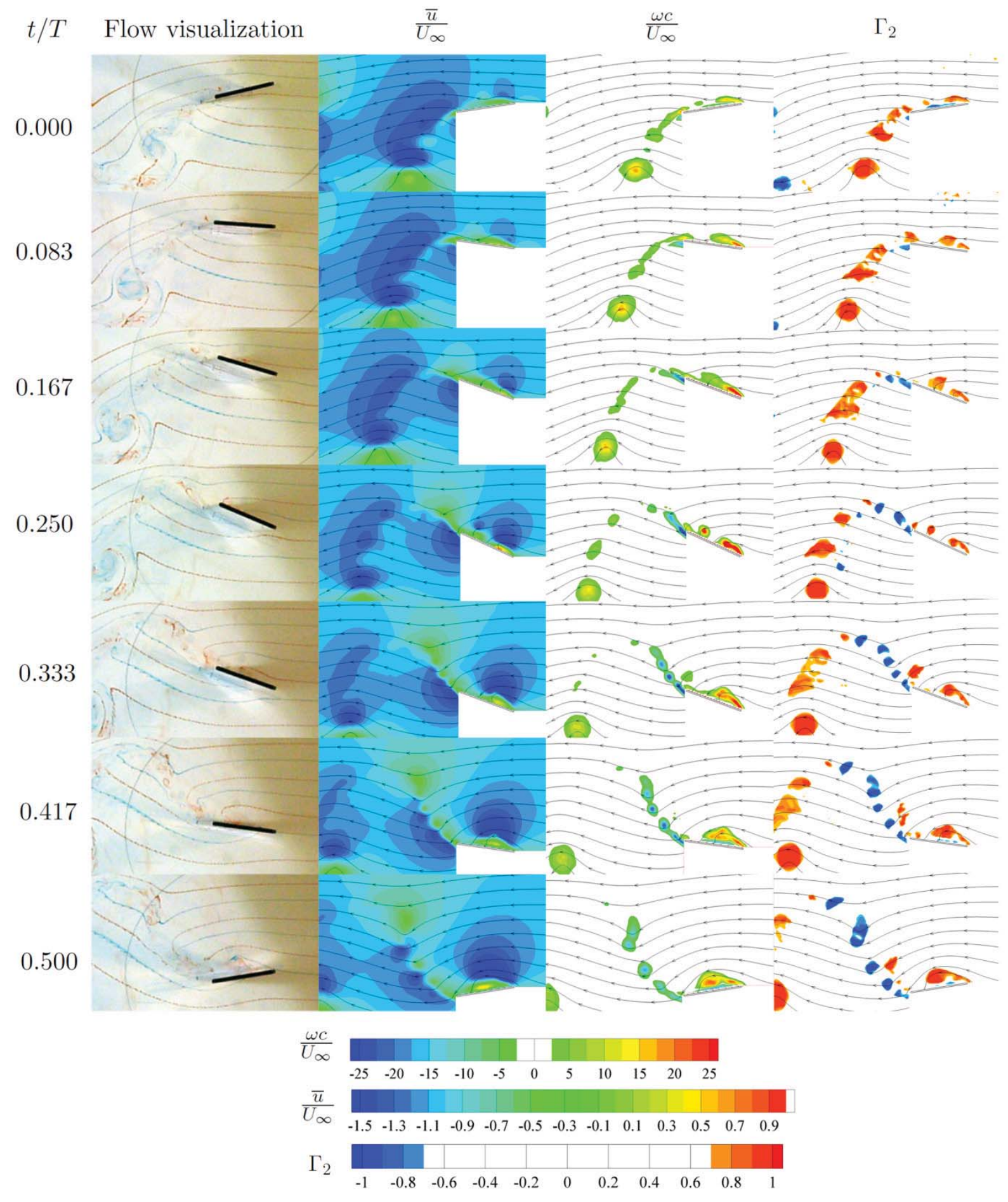

Figure A3. Case 2: Flow visualization, normalized $x$-component velocity contours, normalized vorticity contours at $t / T=$ $0.000,0.083,0.167,0.250,0.333,0.417$, and 0.500 . 


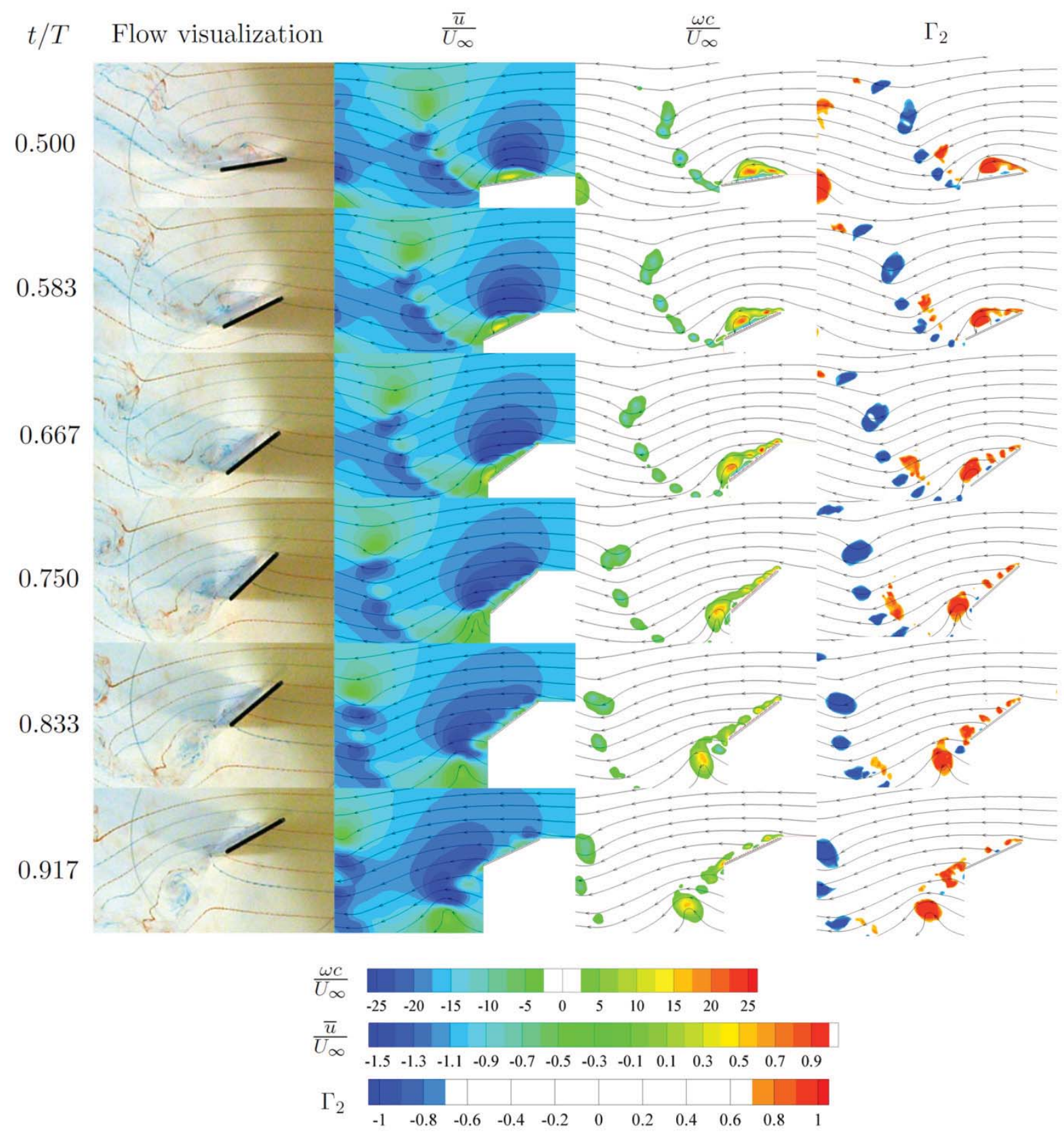

Figure A4. Case 2: Flow visualization, normalized x-component velocity contours, normalized vorticity contours at $t / T=$ $0.500,0.583,0.667,0.750,0.833$, and 0.917 . 


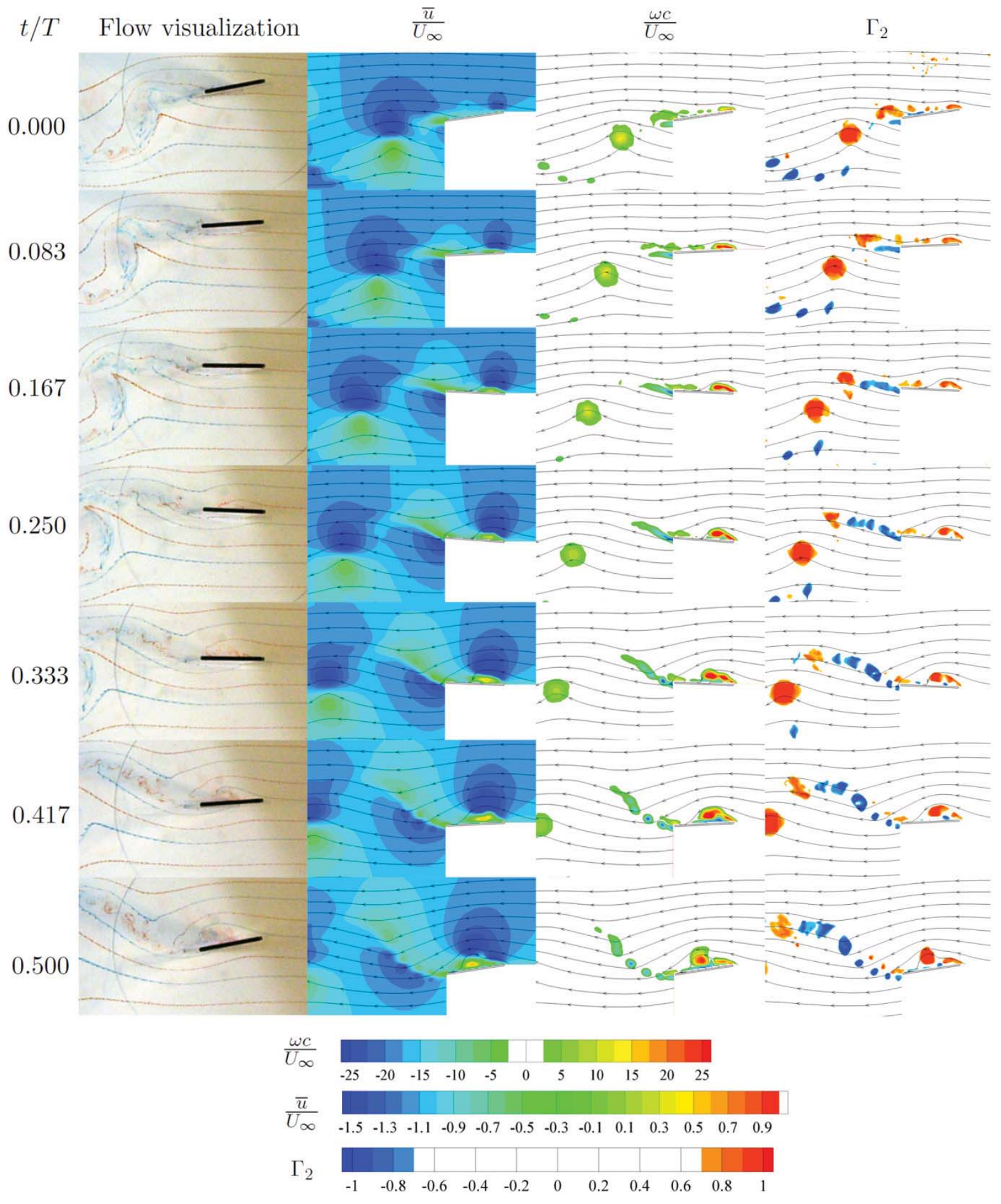

Figure A5. Case 3: Flow visualization, normalized $x$-component velocity contours, normalized vorticity contours at $t / T=$ $0.000,0.083,0.167,0.250,0.333,0.417$, and 0.500 . 


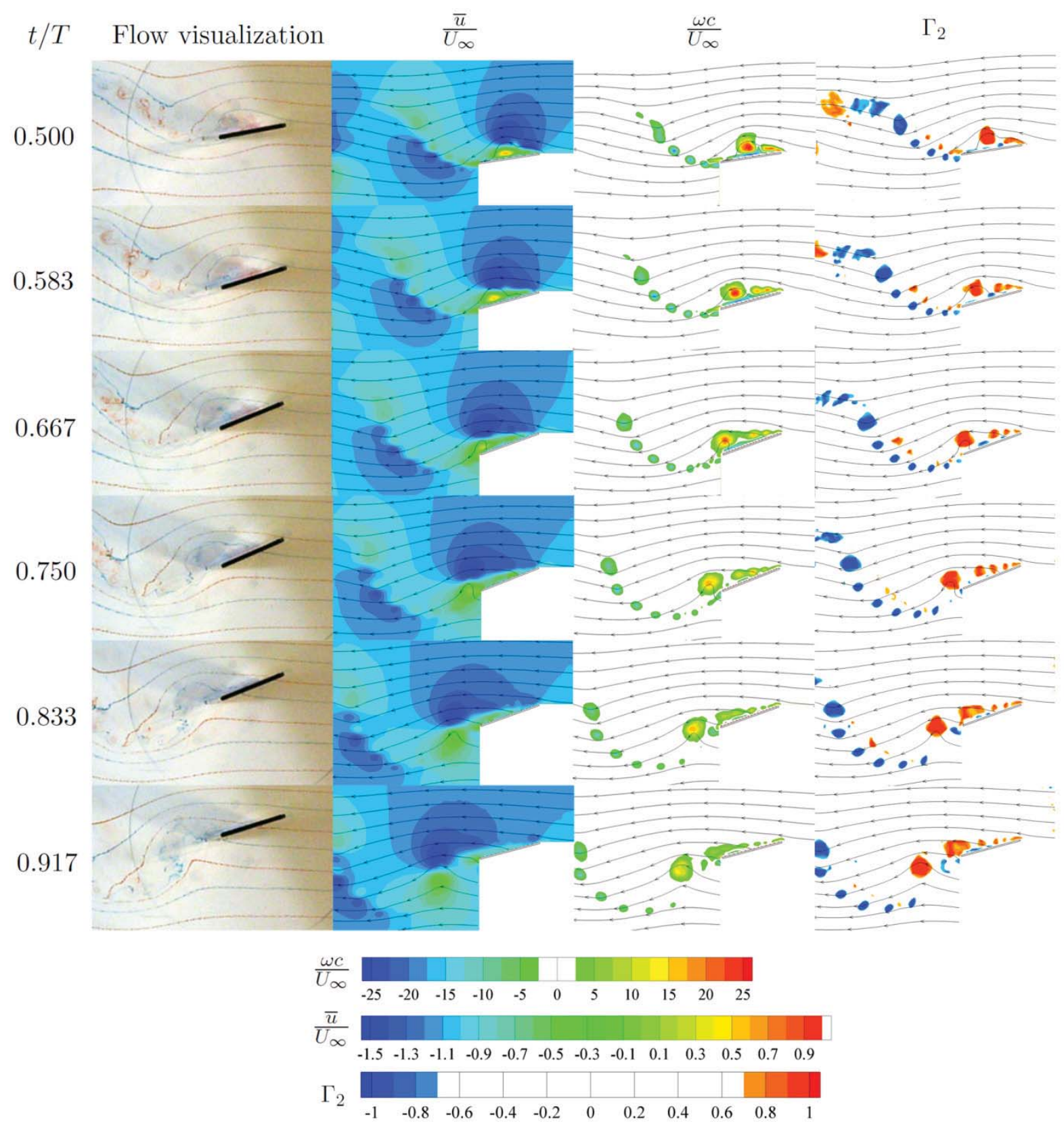

Figure A6. Case 3: Flow visualization, normalized $x$-component velocity contours, normalized vorticity contours at $t / T=$ $0.500,0.583,0.667,0.750,0.833$, and 0.917 . 


\section{References}

[1] Shyy, W., Aono, H., Chimakurthi, S. K., Trizilla, P., Kang, C. K., Cesnik, C. E. S., and Liu, H. (2010). Recent progress in flapping wing aerodynamics and aeroelasticity. Prog Aerosp Sci, Vol. 46, pp. 284-327

[2] Muller, T. J. (2001). Fixed and flapping wing aerodynamics for micro air vehicle applications. AIAA Prog Astronaut Aeronaut, Vol. 195

[3] Pines, D. J., and Bohorquez, F. (2006). Challenges facing future micro-air-vehicle development. J. Aircraft, Vol. 43, pp. 290-305

[4] Shyy, W., Lian, Y., Tang, J., Viieru, D., and Liu, H. (2008). Aerodynamics of low Reynolds number flyers. Cambridge University Press, New York.

[5] Platzer, M., Jones, K., Young, J., and Lai, J. (2008). Flapping Wing Aerodynamics: Progress and Challenges. AIAA J. , Vol. 46, No. 9, pp. 2136-2149.

[6] Triantafyllou, M. S., Triantafyllou, G. S., and Yue, D. K. P. (2000). Hydrodynamics of fishlike swimming. Annu Rev Fluid Mech, Vol. 32, pp. 33-53

[7] Theodorsen, T. (1935). General Theory of Aerodynamic Instability and the Mechanism of Flutter. NACA Report 496

[8] Garrick, I. E. (1936). Propulsion of a Flapping and Oscillating Airfoil. NACA Report 567

[9] McCroskey, W. J. (1981). The phenomenon of dynamic stall. NASA TM-81264 .

[10] Dickinson, M. H., and Gotz, K. G. (1993). Unsteady aerodynamic performance on model wings at low Reynolds numbers. J. Exp. Biol., Vol. 174, pp. 45-64.

[11] Ellington, C. P. (1984). The aerodynamics of hovering insect flight. IV. Aerodynamic mechanisms. Phil. Trans. R. Soc. Lond. B ,Vol. 305, pp. 79-113.

[12] Usherwood, J., and Ellington, C. (2002). The Aerodynamics of Revolving Wings I. Model Hawkmoth Wings. J. Exp. Biol., Vol. 205, pp. 1547-1564.

[13] Ellington, C. P., van den Berg, C., Willmott, A. P., and Thomas, A. L. R. (1996). Leading-edge vortices in insect flight. Nature, Vol. 384, pp. 626-630

[14] van den Berg, C., and Ellington C. P. (1997). The three-dimensional leading-edge vortex of a 'hovering' model hawkmoth. Phil Trans R Soc Lond B, Vol. 352, pp. 329-340

[15] Willmott, A. P., Ellington, C. P., and Thomas, A. L. R. (1997). Flow visualization and unsteady aerodynamics in the flight of the hawkmoth, Manduca sexta. Phil Trans $R$ Soc Lond B, Vol. 352, pp. 303-316

[16] Maxworthy, T. (1979). Experiments on the Weis-Fogh mechanism of lift eneration by insects in hovering flight Part 1. Dynamics of the 'fling'. J. Fluid Mech., Vol. 93, pp. 47-63. 
[17] Shyy, W., and Liu, H. (2007). Flapping wings and aerodynamics lift: the role of leading-edge vortices. AIAA J., Vol. 45, pp. 2817-2819

[18] Gursul, I., and Ho, C. (1992). High Aerodynamic Loads on an Airfoil Submerged in an Unsteady Stream. AIAA J., Vol. 30, pp. 1117-1119

[19] Freymuth, P. (1990). Thrust generation by an airfoil in hover modes. Experiments in Fluids, Vol. 9, pp. 17-24.

[20] Godoy-Diana, R., Aider, J. L., and Wesfried, J. E. (2008). Transitions in the wake of a flapping foil. Phys Rev E, Vol. 77, 016308-1-016308-5

[21] Godoy-Diana, R., Marias, C., Aider, J. L., and Wesfried, J. E. (2009). A model for the symmetry breaking of the reverse Benard-von Karman vortex street produced by a flapping foil. J. Fluid Mech., Vol. 622, pp. 23-32

[22] von Ellenrieder, K. D., and Posthos, S. (2008). PIV measurements of the asymmetric wake of a two dimensional heaving hydrofoil. Exp. Fluids, Vol. 44, pp. 733-745

[23] Lai, J. C. S., and Platzer, M. F. (1999). Jet characteristics of a plunging airfoil. AIAA J., Vol 37, pp. 15291537

[24] Lua, K. B., Lim, T. T., Yeo K. S., and Oo, G. Y. (2007). Wake-structure formation of a heaving twodimensional elliptic airfoil. AIAA J., Vol. 45, pp. 1571-1583

[25] Baik, Y., Rausch, J., Bernal, L. P., Ol, M. V., and Shyy, W. (2010). Experimental Study of Governing Parameters in Pitching and Plunging Airfoil at Low Reynolds Number, AIAA 2010-388

[26] Ol, M. V., Bernal, L. P., Kang, C. K., and Shyy, W. (2009). Shallow and deep dynamic stall for flapping low Reynolds number airfoils. Exp. Fluids, Vol 46, pp. 883-901

[27] Read, D. A., Hover, F.S., and Triantafyllou, M. S. (2003). Effect of angle of attack profiles in flapping foil propulsion. J. Fluid Struct, Vol. 17, pp. 163-183

[28] Hover, F.S., Haugsdal, O., and Triantafyllou, M. S. (2004). Forces on oscillating foils for propulsion and maneuvering. J. Fluid Struct, Vol. 19, pp. 37-47

[29] Schouveiler, L., Hover F. S., and Triantafyllou, M. S. (2005). Performance of flapping foil propulsion, J. Fluid Struct, Vol. 20, pp. 949-959

[30] Taylor, G. K., Nudds, R. L., and Thomas, A. L. (2003). Flying and swimming animals cruise at a Strouhal number tuned for high power efficiency. Nature, Vol. 425, pp. 707-711.

[31] Anderson, J. M., Streitlien, K., Barrett, D. S., \& Triantafyllou, M. S. (1998). Oscillating foils of high propulsive efficiency. J. Fluid Mech., Vol. 360, pp. 41-72.

[32] Baik, Y., Rausch, J., Bernal, L. P. and Ol, M. V. (2009). Experimental Investigation of Pitching and Plunging Airfoils at Reynolds Number between $1 \times 10^{4}$ and $6 \times 10^{4}$, AIAA 2009-4030

[33] Kang, C., Aono, H., Trizila, P., Baik, Y., Rausch, J., Bernal, L. P., Ol, M. V., and Shyy, W. (2009). Modeling of Pitching and Plunging Airfoils at Reynolds Number between $1 \times 10^{4}$ and $6 \times 10^{4}$, AIAA 2009-4100 
[34] Noca, F., Shiels, D., and Jeon, D. (1999). A Comparison of Methods for Evaluating Time-dependent Fluid Dynamic Forces on Bodies, Using Only Velocity Fields and Their Derivatives. . J. Fluid Struct, Vol. 13, pp. 551-578.

[35] Graftieaux, L., Michard, M., and Grosjean, N. (2001). Combining PIV, POD and vortex identification algorithms for the study of unsteady turbulent swirling flows. Measurement Science and Technology, Vol. 12, pp. $1422-1429$

[36] Charkraborty, P., Balachandar, S., and Adrian, R. J. (2005). On the relationship between local vortex identification schemes. J. Fluid Mech., Vol. 535, pp. 189-214 